\title{
Identification of Two Distinct Working Memory- Related Brain Networks in Healthy Young Adults
}

\author{
(1DTobias Egli, ${ }^{1,2}$ (D)David Coynel, ${ }^{2,3}$ (․) Klara Spalek, ${ }^{2,3}$ Matthias Fastenrath, ${ }^{2,3}$ Virginie Freytag, ${ }^{1,2}$ (1) Angela \\ Heck, ${ }^{1,2,4}$ Eva Loos, ${ }^{2,3}$ Bianca Auschra, ${ }^{1,2}$ Andreas Papassotiropoulos, ${ }^{1,2,4,5}$ Dominique J.-F. de \\ Quervain, ${ }^{2,3,4}$ and ${ }^{\circledR}$ Annette Milnik ${ }^{1,2,4}$
}

\section{DOI:http://dx.doi.org/10.1523/ENEURO.0222-17.2018}

\begin{abstract}
${ }^{1}$ Division of Molecular Neuroscience, Department of Psychology, University of Basel, $\mathrm{CH}-4055$ Basel, Switzerland, ${ }^{2}$ Transfaculty Research Platform Molecular and Cognitive Neurosciences, University of Basel, $\mathrm{CH}-4055 \mathrm{Basel}$, Switzerland, ${ }^{3}$ Division of Cognitive Neuroscience, Department of Psychology, University of Basel, $\mathrm{CH}-4055 \mathrm{Basel}$, Switzerland, ${ }^{4}$ Psychiatric University Clinics, University of Basel, $\mathrm{CH}-4055$ Basel, Switzerland, and ${ }^{5}$ Department Biozentrum, Life Sciences Training Facility, University of Basel, CH-4056 Basel, Switzerland
\end{abstract}

\begin{abstract}
Working memory (WM) is an important cognitive domain for everyday life functioning and is often disturbed in neuropsychiatric disorders. Functional magnetic resonance imaging (fMRI) studies in humans show that distributed brain areas typically described as fronto-parietal regions are implicated in WM tasks. Based on data from a large sample of healthy young adults $(N=1369)$, we applied independent component analysis (ICA) to the WM-fMRI signal and identified two distinct networks that were relevant for differences in individual WM task performance. A parietally-centered network was particularly relevant for individual differences in task measures related to WM performance ("WM dependent") and a frontally-centered network was relevant for differences in attention-dependent task performance. Importantly, frontal areas that are typically considered as key regions for WM were either involved in both WM-dependent and attention-dependent performance, or in attentiondependent performance only. The networks identified here are provided as publicly available datasets. These networks can be applied in future studies to derive a low-dimensional representation of the overall WM brain activation.
\end{abstract}

Key words: cognition; functional networks; ICA; n-back; working memory

\section{Significance Statement}

Fronto-parietal brain regions are typically involved when performing working memory (WM) related tasks. Within these fronto-parietal brain regions we have identified two networks that show distinct functional characteristics. Whereas frontal areas are often considered as key regions for WM, we show that frontal areas were either involved in both WM-dependent and attention-related performances or in attentionrelated performance only. A predominately parietally-centered network was the key region for WMdependent performance. Due to the large sample size of $N=1369$ healthy young adults, we can provide robust estimates of these networks which can be applied in future studies.

\section{Introduction}

Working memory (WM) describes the ability to temporarily maintain and manipulate a limited amount of information

Received June 27, 2017; accepted January 18, 2018; First published January 30, 2018.

The authors declare no competing financial interests.
(Baddeley, 2012; Eriksson et al., 2015). It comprises a mental representation of our current environment that can be integrated with previous experiences. Impaired WM leads 
to deterioration in everyday life functioning. Correspondingly WM is affected in neuropsychiatric disorders such as schizophrenia (Lee and Park, 2005; Forbes et al., 2009; Mesholam-Gately et al., 2009; Van Snellenberg et al., 2016), depression (Marazziti et al., 2010), and attentiondeficit hyperactivity disorder (Alderson et al., 2013). Furthermore, white matter microstructure is associated with WM performance and activity in WM related regions (Charlton et al., 2010; Vestergaard et al., 2011; Darki and Klingberg, 2015). In contrast, impairment of white matter integrity comes along with a decrease in WM performance and alterations in the activity of WM-related brain regions (Palacios et al., 2012).

Functional magnetic resonance imaging (fMRI) experiments show that WM-related tasks robustly activate the lateral and medial premotor cortex, dorsolateral prefrontal cortex (DLPFC) and ventrolateral PFC, frontal pole, as well as medial and lateral posterior parietal cortex (Owen et al., 2005; Wager and Smith, 2003; Rottschy et al., 2012). This broad WM network (WMN) of activated brain regions has been studied extensively, including the use of metaanalytical approaches (Yarkoni et al., 2011; Rottschy et al., 2012). Several studies have observed associations of WM performance with mainly parietal or fronto-parietal brain activation (Klingberg et al., 2002; Todd and Marois, 2004, 2005; Nagel et al., 2005; Palacios et al., 2012; Satterthwaite et al., 2013; Zou et al., 2013; Ullman et al., 2014; Darki and Klingberg, 2015; Huang et al., 2016) in children as well as in adults. Recent studies suggest that frontal and parietal regions differ regarding their contributions to WM. Neuronal recordings in the PFC and the lateral intraparietal (LIP) region of monkeys showed that encoded stimuli were retained in both regions, with more task-specific mnemonic encoding in the LIP as compared to the PFC (Sarma et al., 2016). Another study provided causal evidence for differing roles of parietal and frontal regions in attentional aspects of WM processing, by applying transcranial direct current stimulation. Stimulating the right parietal cortex increased the amount of information maintained in the visual WM, whereas stimulating the right PFC improved focusing on relevant information and directing attention away from irrelevant stimuli ( $\mathrm{Li}$ et al., 2017). In addition, measuring the directed connectivity between the DLPFC and superior parietal lobule (SPL) during a visual WM task hinted toward a top-down drive

This work was supported by grants from the Swiss National Science Foundation and the University of Basel.

Acknowledgments: Calculations were in part performed at sciCORE scientific computing core facility at University of Basel (http://scicore.unibas.ch/). We thank Elmar Merkle, Christoph Stippich, and Oliver Bieri for granting access to the fMRI facilities of the University Hospital Basel.

Correspondence should be addressed to either of the following: Tobias Egli, Division of Molecular Neuroscience, University of Basel, Birmannsgasse 8, CH-4055 Basel, Switzerland, E-mail: tobias.egli@unibas.ch; or Annette Milnik, Division of Molecular Neuroscience, University of Basel, Birmannsgasse 8, CH-4055 Basel, Switzerland, E-mail: annette.milnik@unibas.ch.

DOI:http://dx.doi.org/10.1523/ENEURO.0222-17.2018

Copyright (C) 2018 Egli et al.

This is an open-access article distributed under the terms of the Creative Commons Attribution 4.0 International license, which permits unrestricted use, distribution and reproduction in any medium provided that the original work is properly attributed. from DLPFC to SPL that increased with WM load (Kundu et al., 2015). These insights were based on a priori defined regions of interest (ROIs) and therefore described functional properties of separate brain regions.

Importantly, the human brain is organized in functional intrinsic networks that are relatively stable during resting state as well as task execution (Cole et al., 2014; Cole et al., 2016), can exhibit spatial overlaps (Yeo et al., 2014), and are also affected by neurodegenerative diseases (Seeley et al., 2009; Zhou et al., 2012). Hence, instead of applying a ROI-based approach, we used independent component analysis (ICA) to identify distinct networks within the WMN, as measured by the n-back task, based on data from a large $(N=1369)$ sample of healthy young adults. ICA decomposition is a data-driven unbiased approach to retrieve a low-dimensional representation of a dataset, resulting in statistically independent signals (Kong et al., 2008). We included both cortical and subcortical regions into the ICA decomposition to retrieve maximally unbiased estimates of brain networks. To functionally classify these networks, we used cognitive performance measurements of our subjects. We verified the stability of our results using bootstrapping and crossvalidation procedures. Furthermore, we assessed whether microstructural differences of white matter, measured by diffusion tensor imaging (DTI), were associated with activation differences in the estimated networks. Finally, we compared the networks estimated in our study with results from an extensive meta-analysis of neuroimaging studies on WM brain activation (Rottschy et al., 2012) and with networks derived from NeuroSynth, a meta-analytical platform comprising a large variety of different fMRI studies (Yarkoni et al., 2011). All results obtained (univariate statistics and estimates from the ICAs) are available as parametric maps stored on NeuroVault (http://neurovault. org/collections/EYCSLZUZ/; Gorgolewski et al., 2016) and can be used for future studies. The WMN-IC estimates can be used to derive a low-dimensional representation of the overall WM brain activation.

\section{Materials and Methods}

\section{Study and sample description}

We used data from a single-center $\mathrm{fMRI}$ study that aims to identify biological correlates of cognitive performance by combining imaging data with genetics data; note that no genetic data were used here. With respect to the cognitive performance measurements, this study emphasizes on WM and episodic memory performance. The sample consisted of healthy young adults from the general population. We analyzed data of 1369 subjects (mean age: 22.4, range: 18-35; 841 females; the experiment took place at the University Hospital of Basel) after excluding subjects with incomplete behavioral data $(N=$ 28), with cognitive measurements (WM, attention, reaction time, episodic memory, recognition memory) lying 4 SDs above or below the average $(N=15)$, with corrupted imaging data ( $N=38$, see below, $\mathrm{fMRI}$ preprocessing and first-level analyses of the n-back task), or with incomplete imaging data ( $N=6$, see below, fMRl preprocessing and first-level analyses of the n-back task). Subjects were free 
from any neurologic or psychiatric illness and did not take any medication (except oral contraception) at the time of the experiment. Women using hormonal contraceptives (e.g., oral, spiral, patch) and naturally cycling women were included in the study without restrictions. The ethics committees of the Cantons of Basel-Stadt and Basel-Landschaft approved the study. Advertising for study participation was conducted mainly in the University of Basel. Written informed consent was obtained from all subjects before participation.

\section{Experimental procedure}

After receiving general information about the study and giving their written informed consent, participants were first instructed and then trained on a picture-rating task and an $n$-back task. This training was done outside of the MR scanner. After training, participants were positioned in the scanner. All subjects wore earplugs and headphones during MR scans to reduce scanner noise. The participants were instructed not to move during the scans. Small foam pads were used for additional head fixation. We used MR-compatible LCD goggles (VisualSystem, NordicNeuroLab) to present the behavioral tasks inside the scanner. Vision correction was used if necessary. The participants first performed the picture-encoding task in which they had to rate pictures. Afterward they performed the WM task (n-back). During this first fMRI session participants spent a total of $30 \mathrm{~min}$ in the scanner $(20 \mathrm{~min}$ on the picture-rating task, 10 min on the n-back task). Participants then left the scanner and performed an unannounced free recall task of the previously presented pictures (without any time restriction). On finishing the free recall, subjects were instructed and trained on a picture recognition task. This training was done outside of the scanner. Subjects were then positioned in the MR scanner a second time. The picture recognition task lasted 20 min and was followed by T1 (anatomic MRI) and DTI measurements for a further $20 \mathrm{~min}$. The total length of the experimental procedure ranged from 3 to $4.5 \mathrm{~h}$ per subject. Participants were rewarded with 25 Swiss Francs per hour for participating.

\section{WM task description}

We used two different conditions of a verbal n-back task. The 0-back condition required participants to respond to the occurrence of the letter " $x$ " as target stimulus (both lower- and uppercase) in a sequence of letters (e.g., $\mathrm{N}-\mathrm{p}-\mathrm{X}-\mathrm{g}$. . .); all other letters were nontarget stimuli. In the 2-back condition subjects had to indicate whether the current letter and the letter presented two places prior in the sequence were identical (target stimulus) or not (nontarget stimulus); e.g., $S-f-s-g$. . Each condition was measured in six blocks. Every block consisted of 14 stimuli. In each block, three target stimuli and 11 nontarget stimuli were presented (quasi)-randomly; the frequency of lure trials (i.e., the most recent letter matches the letter one or three positions back) was set to $17.9 \%$ (15 out of 84 stimuli) in the 2-back condition. Each block started with an instruction of $5 \mathrm{~s}$ and had a total duration of $33 \mathrm{~s}$. Each stimulus was presented for $500 \mathrm{~ms}$ with a $1500-m s$ interstimulus interval showing a black screen.
The sequence of 2-back and 0-back blocks was randomized and a break of $20 \mathrm{~s}$ was added after every second block. The subjects used a button-box to indicate each stimulus either as "target" or as "nontarget." The data were disregarded if responses were missing (1) in $>30 \%$ of all stimuli across all twelve blocks of the task, (2) in $>30 \%$ of target stimuli in at least three blocks, or (3) in $>30 \%$ of nontarget stimuli in at least three blocks. Task performances were defined as D-prime measures (Macmillan and Creelman, 1990). These measures account for false alarms and were calculated separately for the 0 -back and 2-back conditions. The task performance ranged from -0.34 to $4.34(M=2.53 ; M d=2.47)$ for the D-prime 2-back and from 1.56-4.34 ( $M=3.65$; $M d=$ 3.76) for the D-prime 0-back. We also used the difference in performances between D-prime 2-back and D-prime 0-back, which ranged from -4.10 to $1.38(M=-1.13, M d=$ -1.10). As a measure of difference in reaction times, we used the subtracted reaction time of the two conditions (reaction time 2-back - 0-back), which varied from -37.26 to 602.32 $\mathrm{ms}(M=126.15 \mathrm{~ms} ; M d=104.25 \mathrm{~ms})$.

\section{Descriptions of picture-related tasks}

The picture-rating task required the participants to rate 72 pictures of positive, neutral, and negative valence (24 per valence group). While watching the pictures the participants rated each picture's emotional valence (positive, neutral, negative) and the perceived arousal (low, middle, high) on separate three-point Likert scales. Approximately 10 min later, the subjects were instructed to describe as many of these pictures as possible and in as much detail as possible by using keywords or short sentences (free recall of pictures). Based on these descriptions two independent and blinded raters identified the number of correctly recalled pictures (Cronbachs $\alpha$ between the two raters was 0.91 to 0.98 ). A third independent rater decided on ambiguously scored pictures. The number of correctly recalled pictures served as a measure of episodic memory performance (range: 5-55 pictures; $M=30.77$; $M d=$ 31). This free recall of the pictures was conducted in several different rooms; the effect of the different rooms on the free recall performance was regressed out before running the analyses.

In the picture recognition task, 144 pictures in total were presented: the 72 previously seen pictures and 72 new pictures. The participants rated these pictures as remembered, familiar, or new on a three-point Likert scale. Item familiarity corresponds to the number of previously seen pictures that were identified as "familiar," corrected for the number of new pictures that were wrongly rated as familiar. The item familiarity performance ranged from -32 to $48(M=3.53 ; M d=2)$. Both, the episodic memory task and the familiarity memory task used photographic pictures of positive, neutral, and negative valence selected from the International Affective Picture System (IAPS; Lang et al., 2008). In-house standardized pictures additionally complemented the neutral picture set to equate the stimuli for visual complexity and content (e.g., human presence). 


\section{Description of further task performances and covariates}

A total of $90.3 \%$ of the participants used their right hand while performing the tasks in the scanner, 9.7\% used their left hand. The self-reported body mass index (BMI) ranged from 16.6 to $36.3(M=22.19 ; M d=21.80)$. We assessed distinct chronotypes on a two-point Likert scale: subjects classified themselves either as "eveningness" $(69.8 \%)$ or as "morningness" $(30.2 \%)$ chronotype. The self-reported sleep duration ranged from 3.75 to $12 \mathrm{~h}$ $(M=7.96 ; M d=8)$. Self-reported smoking was measured on a five-point Likert scale ranging from 1 (never) up to 5 (20 cigarettes per day); the relative frequencies per category were: (1) $65 \%$, (2) $23 \%$, (3) $5.2 \%$, (4) $6.8 \%$, (5) $0.7 \%$. After finishing all tasks, the perceived overall task difficulty and the overall motivation of the subjects were measured on five-point Likert scales ranging from 1 (not at all) up to 5 (very). The relative frequencies per category for task difficulty were: (1) $9.2 \%$, (2) $40.3 \%$, (3) $38.1 \%$, (4) $12 \%$, (5) $0.3 \%$ and for motivation were: (1) $0 \%$, (2) $0.5 \%$, (3) $6.6 \%$, (4) $44.4 \%$, (5) $48.4 \%$.

\section{(f)MRI data acquisition}

All functional and structural images were acquired on the same Siemens Magnetom Verio $3 \mathrm{~T}$ whole-body MR unit (12-channel head coil). Blood oxygen level-dependent $\mathrm{fMRI}$ was acquired using a single-shot echoplanar sequence along with generalized auto-calibrating partially parallel acquisition (GRAPPA), using the following parameters: echo time $(\mathrm{TE})=25 \mathrm{~ms}$, field of view $(\mathrm{FOV})=22$ $\mathrm{cm}$, acquisition matrix $=80 \times 80$ (interpolated to $128 \times$ 128 , voxel size $2.75 \times 2.75 \times 4 \mathrm{~mm}^{3}$ ) and with an acceleration factor of 2 . We used an ascending interleaved sequence with repetition time $(\mathrm{TR})=3000 \mathrm{~ms}\left(\alpha=82^{\circ}\right)$ measuring 32 contiguous axial slices that were placed along the anterior-posterior commissure plane based on a midsagittal scout image. A magnetization-prepared rapid acquisition gradient echo T1-weighted image was acquired using the following parameters: TR $=2000 \mathrm{~ms}$, TE $=$ $3.37 \mathrm{~ms}, \mathrm{Tl}=1000 \mathrm{~ms}$, flip angle $=8^{\circ}, 176$ slices, FOV $256 \mathrm{~mm}$, and voxel size $=1 \mathrm{~mm}^{3}$. Automatic segmentations of cortical and subcortical structures were obtained using FreeSurfer 4.5 (v4.5, http://surfer.nmr.mgh.harvard.edu/; RRID:SCR_001847; Fischl, 2012), and labeling was based on the Desikan Atlas (Desikan et al., 2006).

\section{fMRI preprocessing and first-level analyses of the n-back task}

After visual inspection by three raters, 38 participants were excluded due to corrupted T1-weighted images (movement or anatomic abnormalities). MR images were preprocessed with SPM8 (Statistical Parametric Mapping, Wellcome Trust Center for Neuroimaging; http://www.fil. ion.ucl.ac.uk/spm/) implemented in MATLAB R2011b (MathWorks). Slice-time correction to the first slice and realignment were applied using the "register to mean" option. Coregistration of the averaged realigned time series to the structural image ensured spatial alignment of functional and structural images. Subject-to-template normalization was done using DARTEL (Ashburner, 2007), which allows registration to both cortical and subcortical regions and has been shown to perform well in volumebased alignment (Klein et al., 2009). Normalization incorporated the following four steps. (1) Structural images of each subject were segmented using the "New Segment" procedure in SPM8. (2) The resulting gray and white matter images were used to derive a study-specific group template. The template was computed from a subgroup of 1000 subjects (Heck et al., 2014), which were part of the 1369 subjects in the present study. (3) An affine transformation was applied to map the group template to $\mathrm{MNI}$ space. (4) Subject-to-template and template-to$\mathrm{MNI}$ transformations were combined to map the functional images to $\mathrm{MNI}$ space. The functional images were smoothed with an isotropic $8 \mathrm{~mm}$ full-width at halfmaximum (FWHM) Gaussian filter. Intrinsic autocorrelations were accounted for by $\mathrm{AR}(1)$ and low-frequency drifts were removed via high-pass filter (time constant 128 s). Separate regressors were constructed for the 0 - and 2-back conditions comprising a boxcar reference wave form convolved with a canonical hemodynamic response function. Events during the presentation of the instruction as well as movement regressors from spatial realignment were modeled separately. To measure WM-related brain activation we calculated the difference between the 2-back and 0-back parameter estimates for each subject and voxel (first-level 2-back 0 -back contrast). Performance measurements were not included in the first-level analyses.

\section{fMRI group-level analysis}

All further analyses were conducted using the statistical software environment R (3.2.2; RRID:SCR_001905). The 2-back - 0-back contrast parameters from the first-level analyses of $N=1375$ subjects and of $N=71222$ voxels entered the group analyses. Data of six subjects were removed from the analyses because of high numbers of missing voxels ( $>4 \mathrm{SD}$ above average). For the remaining $N=1369$ subjects, we then restricted all analyses to voxels without missing values ( $N=55,614$ voxels). Based on one-sample $t$ tests, we identified all voxels that were more active in the 2-back in comparison to the 0-back condition when applying FDR correction $(\alpha=5 \%)$.

Across the timespan of the data acquisition, the gradient coils were changed twice (hardware batches), and parts of the scanner's software configuration were changed once (software batches). Additionally, the scanner console displayed irregularities during the data acquisition in a small group of subjects (processing batches). We regressed out these potential groupeffects from the voxel-signal; we used the standardized residuals to perform the ICA decomposition and the association analyses.

\section{Identification of distinct WMN subnetworks by using ICA decomposition}

We investigated the distribution of 2-back - 0-back contrast parameter estimates by measuring the skewness, kurtosis, and Shapiro-Wilk tests. The data were highly skewed across subjects $(-2.59-2.34)$ and voxel (-2.03-2.84), showed a high kurtosis across subjects (2.98-49.11), and voxel (3.26-23.06) and deviated con- 
siderably from normal distribution across subjects (Shapiro-Wilk test: range $W, 0.85-1.00$; range $-\log _{10}(p), 0.33-$ 33.72) and voxel (Shapiro-Wilk test: range $W, 0.80-1.00$, range $\left.-\log _{10}(p), 6.31-60.60\right)$. Because of the strong nonGaussian components of the 2-back - 0-back contrast parameters, we used ICA as dimensionality reduction method. Applied to a matrix $X$ of $m$ observations (subjects) and $n$ variables (voxels), ICA estimates a matrix of $\mathrm{k} \times \mathrm{n}$ latent sources $\mathrm{S}$ that underlie the variables, holding the source estimates (referred to as voxel loadings throughout the paper) as statistically independent from each other as possible (Engreitz et al., 2010). In addition to the source estimates, ICA also yields a matrix of $m \times k$ mixing coefficients $A$ (referred to as subjects scores throughout the paper) for each IC. The mixing coefficients of a particular component depict the projection of the original data onto this component's estimated source, such that $X=A S$ (Hyvärinen and Oja, 2000). By applying ICA decomposition to a matrix of 2-back - 0-back contrast estimates, containing rows of voxels and columns of subjects, our source estimates (voxel loadings) described statistically independent latent sources that underlie the contrast estimates. Accordingly, each component's mixing coefficients described the activity strength of each component for each subject (Chiappetta et al., 2004). Subjects with high-contrast estimates in the voxels that load highly onto a particular IC in the positive direction obtained elevated scores for this IC. Hence, we interpreted the subject scores as a measure of coactivation in the voxels that loaded onto the IC.

We first applied PCA to determine the number of components to be extracted by the ICA. After visually inspecting the scree plot of the Eigenvalues we decided to retrieve six components. We performed ICA to retrieve these six ICs using the fastlCA algorithm (R-package "fastICA"; Hyvärinen and Oja, 2000) with centering and scaling of the variables as well as applying a PCA and whitening of the data. Since the direction of ICA estimates is arbitrary, we recoded all estimated ICs with the result that the voxels with the highest absolute loadings displayed positive loadings. We retained the source estimates ("voxel loadings") and mixing coefficients ("subject scores") of the extracted ICs (WMN-ICs) for further analyses. Accordingly, every voxel exhibited a voxel loading for each of the six WMN-ICs. For visualization purpose and for anatomic annotation, we determined the voxel loadings with the $10 \%$ most extreme absolute values $(|z|>$ 1.47), when considering all six ICs. All association analyses were conducted on unthresholded WMN-ICs.

\section{Cortical and subcortical labeling of the WMN-ICs}

Labeling of gray matter brain regions was based on a population-averaged probabilistic atlas. The atlas comprises a total of $N=87$ distinct cortical and subcortical brain regions from both hemispheres. Each of the $N=$ 55,614 voxels was assigned to one of these anatomic brain regions. Voxels for which the probability to belong to a given brain region was below $25 \%(N=2926)$ or that were not located within cortical or subcortical regions $(N=21,451)$ were excluded, resulting in $N=31,237$ voxels used for anatomic labeling. For each WMN-IC, we grouped voxels that showed the $10 \%$ most extreme values (see above) into clusters of adjacent voxels (WMN-IC clusters). Within each WMN-IC cluster and for each anatomic brain region, we determined the absolute number of voxels that belonged to this cluster and were annotated with this region. We report only brain regions comprising $>10$ voxels of a WMN-IC cluster. We also calculated the percentage of voxels per $\mathrm{WMN}-\mathrm{IC}$ cluster and anatomic brain region by dividing the absolute number of voxels by the total number of voxels labeled with the anatomic brain region across the $N=31,237$ voxels.

The used population-average probabilistic anatomic atlas was built by automatic gray matter segmentation of the subjects' T1-weighted images. Each participant's T1weighted image was first automatically segmented into cortical and subcortical structures using FreeSurfer (v4.5, http://surfer.nmr.mgh.harvard.edu/; RRID:SCR_001847; Fischl, 2012). Labeling of the cortical gyri was based on the Desikan-Killiany Atlas (Desikan et al., 2006), yielding 35 regions per hemisphere. We also labeled 17 subcortical regions, following Fischl et al., (2002). The segmented T1 image was then normalized to the study-specific anatomic template space using the subject's previously computed warp field, and affine-registered to the MNI (Montreal Neurologic Institute) space (see above, fMRI preprocessing and first-level analyses of the n-back task). Nearest-neighbor interpolation was applied, to preserve labeling of the different structures. The normalized segmentations were finally averaged across subjects, to create a population-average probabilistic atlas. Each voxel of the template could consequently be assigned a probability of belonging to a given anatomic gray matter-segmented structure, based on the information of $N=1000$ subjects that are part of the samples included in this study.

\section{Association with task performance measures}

We assessed the associations of each WMN-IC with performance measurements of multiple behavioral tasks and several covariates using a multiple linear regression model for each WMN-IC. For each WMN-IC, the scores per subject were used as the dependent variables. The task performance measurements and covariates were assigned as independent variables. To reduce multicollinearity between the independent variables and covariates, we excluded strongly correlated variables $\left(\left|r_{\text {Pearson }}\right|>0.5\right)$.

The following behavioral task performances were included: (1) n-back performances (D-prime 2-back; D-prime 0-back), (2) n-back reaction time (difference between reaction times during 2-back condition and 0-back condition), (3) episodic memory, and (4) item familiarity. We first calculated linear models with the difference in 2-back and 0 -back performances as a single predictor. To estimate the associations with 2-back and 0-back performances individually, we also included both performance measurements separately in the model. We further included the following covariates in the analyses: (5) Sex, (6) age at the time of investigation, (7) hand used for task performance, (8) motivation, (9) perceived task difficulty, (10) smoking behavior, (11) usual sleep duration, (12) chronotype, and 
(13) BMI. Since the scores of the WMN-ICs were correlated $\left(r^{2}<0.11\right.$; see results section "Identification of distinct WM-task networks"), we additionally included the scores of the five remaining ICs as covariates in all analyses. The regression models thus comprised 18 predictors when including the difference in 2-back and 0-back performances as a single predictor, and 19 predictors when including separate predictors for 2-back and 0-back performances.

To retrieve standardized regression coefficients (subsequently referred to as regression coefficient or $\beta$ ), all variables were $z$-transformed. By including all predictors and covariates in one linear model, we estimated the association between each variable and the $\mathrm{WMN}-\mathrm{IC}$ while keeping all other included variables constant. Testing of significance for the behavioral task performances was conducted using $t$ tests. We report FDR-corrected $p$ values for associations of WMN-ICs with task performance $(\alpha<5 \%$, correcting for 108 tests based on 18 predictors $\times$ six WMN-ICs with the difference in 2-back and 0-back performances as a single predictor; correcting for 114 tests based on 19 predictors $\times$ six WMN-ICs with 2-back and 0-back performances as separate predictors).

We used the same linear models, but without including the WMN-IC scores as covariates, to estimate the univariate association of each voxel with D-prime 2-back and D-prime 0-back performances. We applied FDR correction $(\alpha=5 \%)$ to account for 371588 independent statistical tests, based on 14 predictors $\times 26542$ voxels.

\section{ICA bootstrapping}

We assessed the stability of the WMN-ICs and of their associations with behavioral measures using a bootstrapping approach. We repeated the following procedure 100 times for two different sizes of the subsamples $N_{\text {subsample }}=$ $[100,684]$. We (1) randomly divided the sample into two subsamples of sizes $N_{\text {subsample }}$ (sampling without replacement, no intersection between the subsamples); (2) for both subsamples, we estimated six ICs; and (3) calculated linear models of the IC estimates against behavioral measures and covariates as described above; and (4) for each IC of both subsamples, we identified the best-matching IC of the total sample. We correlated the source estimates (i.e., voxel-loadings) of these matched ICs from the two subsamples.

\section{ICA cross-validation}

We projected the information from WMN-ICs that were estimated across $N=1269$ subjects onto smaller groups of $N=100$ subjects. We repeated the following procedure 100 times: in each run, (1) we randomly divided the sample into the larger and the smaller subsamples; (2) we estimated six ICs from the WMN in the larger subsample; (3) the ICA estimates were then projected onto the 2-back - 0-back contrast estimates of the smaller subsample; and (4) the resulting projected scores of WMN-IC3 and WMN-IC4 were then regressed against behavioral task performances (D-prime 2-back, D-prime 0-back) and covariates (sex, age) in the smaller subsample. This yielded the percentage of runs in which the projected scores showed significant $\left(p_{\text {nominal }}<0.05\right)$ associations with task performance measures. To retrieve empirical $p$ values for the cross-validation, we repeated the 100 cross-validations 1000 times, after permutation of the task performance measurements. We used the percentages of associations between projected scores and permuted performance measurements as a null distribution.

\section{Association of the WMN-ICs with white matter micro- structure}

Diffusion volumes were acquired for a subset of $N=$ 657 subjects using a single-shot EPI sequence, and consisted of 64 diffusion-weighted volumes with $b=900$ $\mathrm{s} / \mathrm{mm}^{2}$ and one unweighted volume $(\mathrm{b}=0)$. We used the following acquisition parameters: TR $=9 \mathrm{~s}$; TE $=82 \mathrm{~ms}$; FOV $=320 \mathrm{~mm}$; GRAPPA R $=2.0$; voxel size $=2.5 \times$ $2.5 \times 2.5 \mathrm{~mm}^{3}$. Two participants were excluded due to excessive movement during the DTI acquisition. Diffusionweighted images were analyzed using FSL (4.1.7; RRID: SCR_002823; Jenkinson et al., 2012). Images were coregistered to the reference unweighted volume $(b=0)$ using an affine transformation for correction of head motion and eddy current induced image distortion. Maps of fractional anisotropy (FA) were obtained from the diffusion tensor model for further analyses. FA is an estimate of the directional dependence of diffusion (Basser, 1995). It reflects aspects of white matter microstructure that are related to fiber orientation (Jones et al., 2013) and can be modulated by myelination (Beaulieu, 2002). We obtained 70 cortical white matter-segmented regions (35 regions per hemisphere) from the FreeSurfer v4.5 wmparc files. Anatomic labels for the white matter segmentations corresponded to the labels of gray matter segmentations adjacent to the corresponding white matter segmentation. We used the averaged FA values per region for the following analyses. Sixteen participants were excluded due to missing FA measures in any of the white mattersegmented brain regions. Complete datasets (behavior and imaging) were available for $N=614$ participants. For each white matter-segmented brain region and each WMN-IC, we calculated linear regression models with the WMN-IC's scores per subject as dependent variables and the FA estimate as independent variable. Sex, age, handedness, intracranial volume, and scores of the remaining WMN-ICs were used as covariates. We tested separately for each WMN-IC whether the $p$ values of the associations between the $70 \mathrm{FA}$ values and WMN-IC scores deviate from the uniform distribution that is expected for continuous data under a simple null hypothesis (Murdoch et al., 2008). The resulting $p$ values were FDR corrected for six tests $(\alpha<0.05)$. We additionally calculated empirical $p$ values based on the number of nominally significant associations for each WMN-IC after permuting the WMN-IC scores 10000 times, applying FDR correction $(\alpha<0.05)$ for six independent tests.

\section{Description and analysis of the NeuroSynth database}

NeuroSynth is a publicly available database currently comprising data from $11406 \mathrm{fMRI}$ studies summarized in 3107 fMRI meta-analyses for commonly used terms (RRID:SCR_006798; Yarkoni et al., 2011). We obtained the NeuroSynth data files (database.txt; features.txt; ver- 
sion 0.6, released July, 2015) as well as the reverse inference maps of all 3107 meta-analyses. The reverse inference maps of the meta-analyses describe for each voxel the probability of the term being used in the available studies given the activations in the voxel across the studies; these inference maps contain estimates for voxels showing FDR-corrected ( $\alpha=0.01)$ significant associations. We first selected all terms that were reported in at least 250 studies at a high frequency ( $>1$ in 1000 words). For these terms we filtered for all reverse inference maps that comprise at least 1200 FDR-corrected significant voxels (out of 228,453 voxels, $>0.5 \%$; voxel size $2 \times 2 \times$ $2 \mathrm{~mm}$ ). After applying these filter-steps we used the metaanalytic results of 233 terms for the further analyses. We applied z-transformation to the probability estimates for each term before applying PCA. After visually inspecting the scree plot of the PCA (see results section "Comparison of the WM-task networks with external datasets"), we decided to extract 16 components. After whitening of the data we applied ICA decomposition on the probability estimates using the fastlCA algorithm (R-package fastICA; Hyvärinen and Oja, 2000) to retrieve 16 networks that were based on the results of the 233 meta-analyses. Since the direction of ICA estimates is arbitrary, we recoded all estimated ICs with the result that the voxels with the highest absolute loadings displayed positive loadings. The mixing coefficients (score per term) were used to characterize each component (NeuroSynth IC-topic).

The uncorrelated and statistically independent source estimates (loadings per voxel) were coregistered to the image space of our functional MRI data by applying affine transformation with NiftyReg (http://cmictig.cs.ucl.ac.uk/ wiki/index.php/NiftyReg; RRID:SCR_006593; Modat et al., 2010). We tested the overlap between the 16 NeuroSynth networks and the WMN derived from our functional MRI data by calculating the percentage of voxels that show high loadings on the NeuroSynth networks $(|z|>0.70$; i.e., the $10 \%$ most extreme absolute values across all NeuroSynth ICs; the same threshold was used to visualize the NeuroSynth ICs) and were additionally located in the WMN. Furthermore, we compared the loadings per voxel between the NeuroSynth networks and the WMN-ICs (shared variance $r^{2}$ ). We retrieved subject-wise scores for the NeuroSynth IC-topics in our study sample by projecting the NeuroSynth ICA estimates onto the 2-back 0 -back contrast parameter estimates of our subjects. The projected scores for the NeuroSynth IC-topic were regressed against the subjects' task performance measures using multiple linear regression models (including sex, age, hand used for the task, motivation, perceived task difficulty, smoking behavior, usual sleep duration, chronotype and $\mathrm{BMI}$ as covariates). The resulting $p$ values were FDR corrected ( $\alpha=0.05$ ) for 224 independent tests, based on 14 predictors $\times 16$ NeuroSynth ICs.

\section{Brain images}

Figures of clustered voxels within a semitransparent brain (MNI 152 template) were produced using MRIcroGL (http://www.mccauslandcenter.sc.edu/mricrogl/; RRID: SCR_002403) after smoothing (3 mm smoothing kernel) using the R-packages "fslr" (Muschelli et al., 2015) and "oro.nifti" (Whitcher et al., 2011). All brain images are displayed within the MNI152 template and according to neurologic convention (left hemisphere displayed on the left side).

\section{Data repository}

Parametric maps of the main findings (group-activation $t$ values for the 2-back - 0-back contrast parameter; $\beta$ values for associations between the 2-back - 0-back contrast parameters and the 2-back as well as the 0-back performances; $z$ values describing voxel loadings of the ICs) are stored online in the public repository NeuroVault (RRID:SCR_003806; Gorgolewski et al., 2016) and can be retrieved for use in future studies (http://neurovault.org/ collections/EYCSLZUZ/).

\section{Results}

We used two different conditions of a verbal n-back task. The 0-back condition required participants to respond to the occurrence of the letter $x$ (both lower- and uppercase) in a sequence of letters (e.g., $\mathrm{N}-\mathrm{p}-\mathrm{X}-\mathrm{g}$. . .). This control condition requires very low WM load and was used as a measure of attention. In the 2-back condition subjects had to indicate whether the currently presented letter and the letter two places prior in the sequence were identical or not (e.g., $S-f-s-g$. . .). This condition requires online monitoring, updating, and manipulation of remembered information and is therefore assumed to involve key WM-related processes (Owen et al., 2005). Task performances were defined as D-prime measures (Macmillan and Creelman, 1990) that account for false alarms, calculated separately for the 0-back and 2-back conditions. Both behavioral measurements were correlated with a medium effect size $\left(r_{\text {Pearson }}=0.35 ; 12 \%\right.$ shared variance). The 0 -back performance is also referred to as "attention-related" and the 2-back performance is also referred to as "WM-related" task performance in the following sections.

\section{fMRI group-level analysis of the WM-task activation}

The fMRI analyses were based on the 2-back - 0-back contrast parameter estimates. We first applied voxel-wise ( $N=55614$ voxels) one-sample $t$ tests to the contrast parameter estimates. Here, due to the large sample size $(N=1369)$, the whole-brain signal was virtually separated into voxels that were more active in the 2-back condition, and voxels that were more active in the 0 -back condition (see " $t$ value contrast 2-back - 0-back" in NeuroVault). The WMN is typically defined as voxels that are more active in the 2-back condition in comparison to the 0-back condition (Rottschy et al., 2012); the 0-back condition is included to control for sensory-motor processes and attention (Miller et al., 2009). The WMN identified with our data were defined as the 2-back positive voxels of the 2-back - 0-back contrast parameter estimates (whole brain FDR-corrected $\alpha<5 \%$; $N=26,542$ voxels; Fig. 1A). This WMN comprised most of the FDR-corrected metaanalytic result for the term "working memory" acquired from NeuroSynth (Yarkoni et al., 2011; Fig. 1B): 98\% of the WMN voxels derived from NeuroSynth were located within the WMN obtained from our data. 


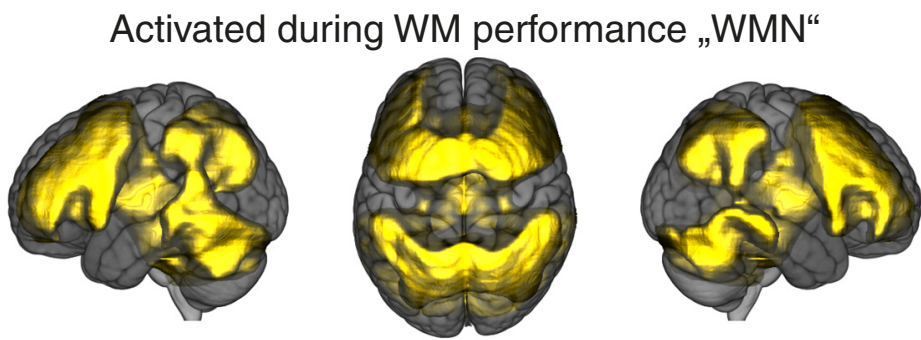

b

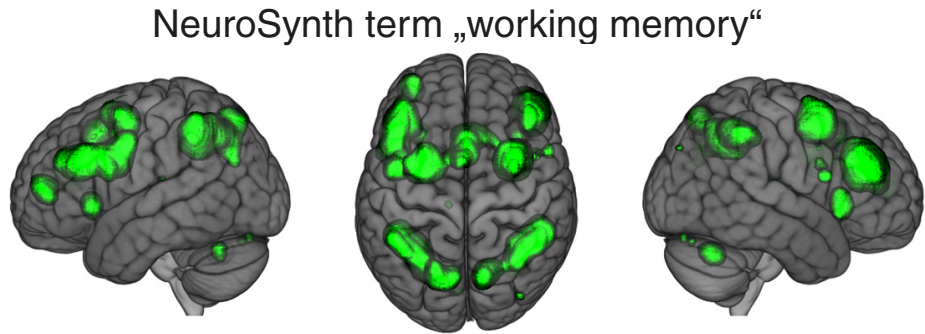

Figure 1. WMNs. A, Brain regions that were more strongly activated during the 2-back condition in comparison to the 0-back condition in our sample (2-back - 0-back contrast one-sample $t$ tests FDR corrected, $\alpha=0.05)$. $\boldsymbol{B}$, Meta-analytic results for the term working memory retrieved from NeuroSynth (reverse inference, FDR corrected, $\alpha=0.01$ ). The brain images are displayed within the MNI152 template and according to neurologic convention.

\section{Identification of distinct WM-task networks}

To identify separable networks of brain activation within the WMN we applied ICA as a dimensionality reduction method. ICA decomposition is a data-driven unbiased approach that models observations as a linear combination of latent components (Engreitz et al., 2010), which are as statistically independent and uncorrelated as possible (Hyvärinen and Oja, 2000). We applied ICA onto the 2-back - 0-back contrast estimates of our subjects. Each voxel obtained one loading per IC, and each subject obtained one score per IC. The ICs were statistically independent and uncorrelated with regard to their voxel loadings. Accordingly, a voxel's loading in a particular IC did not yield any information regarding this voxel's loading in any other IC. When illustrating the voxel loadings of the ICs, we concentrated on the voxels with the most extreme
$10 \%$ of loadings. Whenever a subject showed increased activation in the brain regions that loaded highly onto an IC in the positive direction, the subject received an elevated positive score for the specific IC. Accordingly, the subject scores of an IC represented a measure of coactivation across the voxels that loaded onto this IC. We therefore interpreted the estimated ICs as networks of coactivated brain regions.

Whitening of the data was done based on a principal component analysis (PCA) before applying the ICA. After visually inspecting the Eigenvalues of the PCA (Fig. 2A) we decided to extract six ICs from the WMN ("WMN-ICs"; Fig. 2B). Each WMN-IC was functionally annotated using multiple linear regression models including both D-prime 2-back and D-prime 0-back performances as well as further covariates as independent variables (Table 1; Ex- a

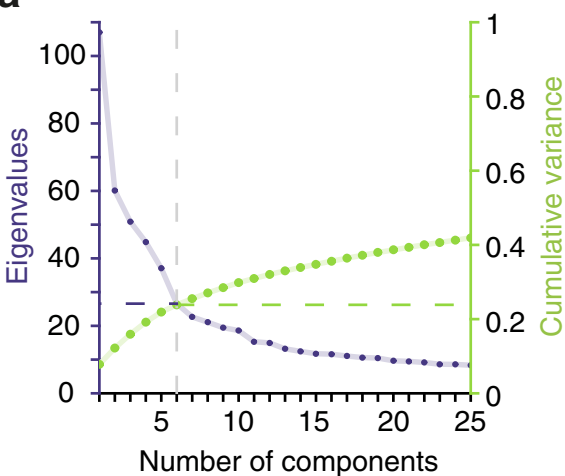

b

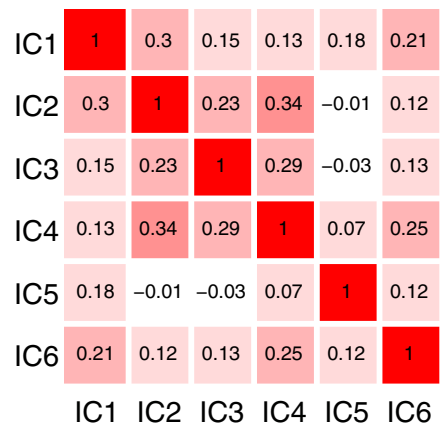

C

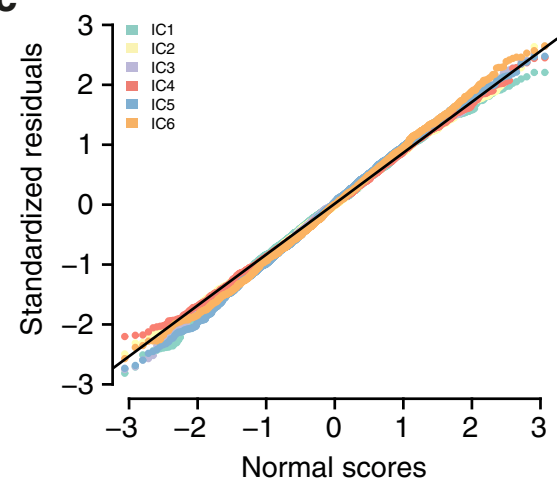

Figure 2. WMN ICA decomposition auxiliary information. $\boldsymbol{A}$, The eigenvalues (purple, left $y$-axis) and cumulative variance (green, right $y$-axis) of a PCA on the WMN. B, Pearson's correlations between WMN-ICs on the subject-level $(N=1369)$. $\boldsymbol{C}$, Quantile-quantile plots comparing the standardized residuals ( $y$-axis) from multiple linear regression models of each WMN-IC against behavioral measurements and covariates (including the remaining WMN-ICs) with a normal distribution ( $x$-axis); D-prime 2-back and D-prime 0-back performances were included as separate predictors in these models. Models with the performance difference of D-prime 2-back and D-prime 0-back as a single predictor yielded highly similar residuals (all $r_{\text {Pearson }}>0.98$ ). 
Table 1. Associations of WMN-ICs with performances

\begin{tabular}{|c|c|c|c|c|c|c|c|c|c|c|}
\hline \multirow[b]{2}{*}{ IC\# } & \multicolumn{2}{|c|}{$\begin{array}{c}\text { D-prime } \\
\text { 2-back - 0-back } \\
(\mathrm{df}=1350)\end{array}$} & \multicolumn{2}{|c|}{$\begin{array}{c}\text { D-prime } \\
\text { 2-back } \\
\text { (df = 1349) }\end{array}$} & \multicolumn{2}{|c|}{$\begin{array}{c}\text { D-prime } \\
\text { 0-back } \\
\text { (df = 1349) }\end{array}$} & \multicolumn{2}{|c|}{$\begin{array}{l}\text { Reaction time } \\
\text { 2-back - 0-back } \\
(\text { df }=1349)\end{array}$} & \multicolumn{2}{|c|}{$\begin{array}{l}\text { Episodic memory } \\
\quad(\mathrm{df}=1349)\end{array}$} \\
\hline & $\beta$ & $p$ & $\beta$ & $p$ & $\beta$ & $p$ & $\beta$ & $p$ & $\beta$ & $p$ \\
\hline IC1 & 0.01 & 0.85 & 0.02 & 0.55 & 0.03 & 0.35 & -0.01 & 0.85 & 0.05 & 0.12 \\
\hline IC2 & 0.01 & 0.69 & -0.01 & 0.74 & -0.07 & $0.02 *$ & -0.02 & 0.66 & 0.08 & $0.006 *$ \\
\hline IC3 & 0.24 & $2.3 \times 10^{-19_{* * *}}$ & 0.24 & $2.8 \times 10^{-18_{* * *}}$ & -0.15 & $7.6 \times 10^{-7} * * *$ & 0.09 & $0.003 *$ & 0.02 & 0.66 \\
\hline IC4 & -0.13 & $2.8 \times 10^{-6_{* * *}}$ & -0.06 & 0.09 & 0.25 & $1.8 \times 10^{-19_{* * *}}$ & -0.02 & 0.52 & -0.04 & 0.18 \\
\hline IC5 & -0.01 & 0.75 & -0.01 & 0.73 & 0.01 & 0.85 & -0.06 & 0.09 & -0.05 & 0.19 \\
\hline IC6 & -0.05 & 0.14 & -0.04 & 0.27 & 0.06 & 0.12 & 0.01 & 0.81 & -0.10 & $9.0 \times 10^{-4} * *$ \\
\hline
\end{tabular}

The reported $p$ values are FDR corrected (see Materials and Methods); $* p<0.05, * * p<0.001, * * * p<0.0001$

The results of the linear models with the WMN-ICs as dependent variables for n-back D-prime performances, n-back reaction time, and episodic memory performance. For the remaining covariates, see Extended data Table 1-1. Voxel-wise associations are described in Extended data Table 1-2. The estimates of statistical power for a voxel-wise analysis and an analysis using WMN-ICs are displayed in Extended data Table 1-3.

tended data Table 1-1; for the distributions of the residuals of the models, see Fig. $2 C$ ).

Two of the six components were associated with the difference of WM-related and attention-related performances (D-prime 2-back - D-prime 0-back; Table 1). WMN-IC3 was positively associated with the performance difference $\left(p_{F D R}=2.3 \times 10^{-19}, R^{2}=0.06\right)$ and WMN-IC4 was negatively associated with the performance difference $\left(p_{F D R}=2.8 \times 10^{-6}, R^{2}=0.02\right)$. We next calculated multiple linear regression models with WM-related performance and attention-related performance as separate predictors. These models were used as main models for all subsequent analyses. In these analyses, WMN-IC3 was significantly associated with both the $\mathrm{D}$-prime 2-back performance $\left(p_{F D R}=2.8 \times 10^{-18}\right.$; Fig. $\left.3 B\right)$ and the D-prime 0-back performance with opposite direction of effects $\left(p_{F D R}=7.6 \times 10^{-7}\right)$. WMN-IC3 explained $5.8 \%$ variance of $D$-prime 2-back performance, $2.2 \%$ variance of $D$-prime 0 -back performance and $0.8 \%$ variance of the difference in reaction time between 2-back and 0-back. This component exhibited the most extreme positive loadings $(z>1.47$, describing the most extreme $10 \%$ of absolute values across the WMN-ICs) in bilateral parietal regions, the bilateral middle frontal gyrus, as well as the left precentral gyrus and pars opercularis (Fig. 3A; Extended data Fig. 3-1). WMN-IC3 was also associated with $\operatorname{sex}\left(p_{F D R}=4.0 \times 10^{-6}\right)$; separate analyses for each gender yielded similar results for WM-related and attention-related performances (males: $N=528,2$-back performance $R^{2}=0.04, p_{F D R}=4.3 \times 10^{-5}$, 0-back performance $R^{2}=0.03, p_{F D R}=4.0 \times 10^{-4}$, opposite directions of effect; females: $N=841,2$-back performance $R^{2}=0.07, p_{F D R}=3.4 \times 10^{-13}, 0$-back performance $R^{2}=0.02, p_{F D R}=0.005$, opposite directions of effect). WMN-IC4 was markedly associated with D-prime 0-back performance $\left(p_{F D R}=1.8 \times 10^{-19}, R^{2}=0.06\right.$; Fig. $3 D)$ but not with $D$-prime 2-back performance $\left(p_{F D R}=\right.$ $\left.0.09, R^{2}=0.004\right)$. This component exhibited main posi- a
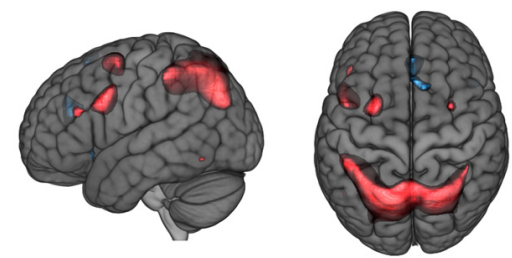

Voxel loadings WMN-IC4

C

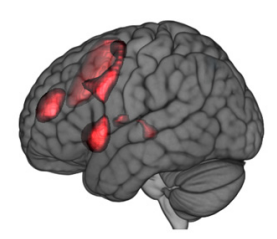

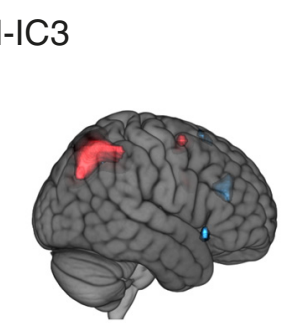

Voxel loadings WMN-IC3

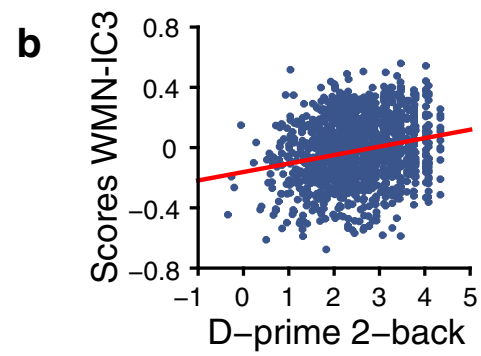

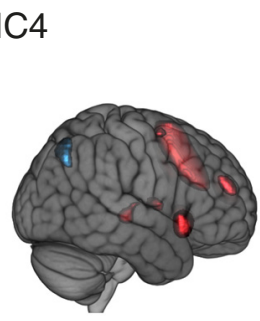
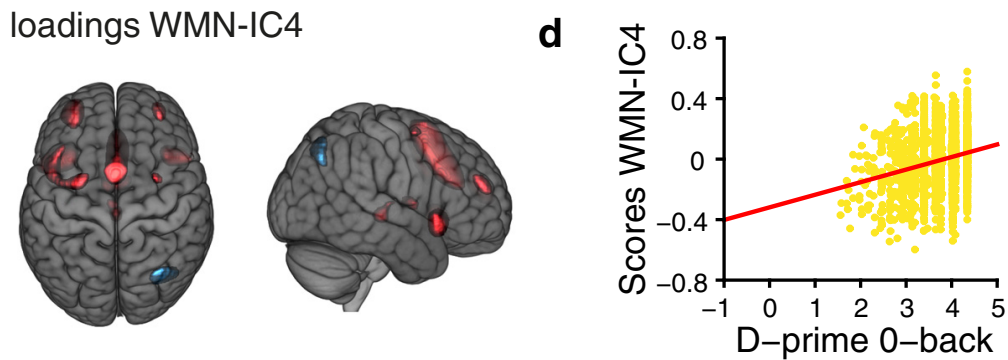

Figure 3. WMN ICA decomposition main findings. Voxel loadings $(\boldsymbol{A})$ of WMN-IC3 and $(\boldsymbol{C})$ of WMN-IC4 illustrated for $|z|>1.47$ showing the most extreme $10 \%$ of the voxel loadings across all WMN-ICs; red depicts positive and blue negative voxel loadings. Associations (B) of WMN-IC3 with D-prime 2-back and (D) of WMN-IC4 with D-prime 0-back task performances. Annotations of WMN-ICs with anatomic regions are listed in Extended data Figure 3-1. The results of additional WMN ICA decompositions with varying numbers of components are illustrated in Extended data Figures 3-2, 3-3. The brain images are displayed within the MNI152 template and according to neurologic convention. 
a

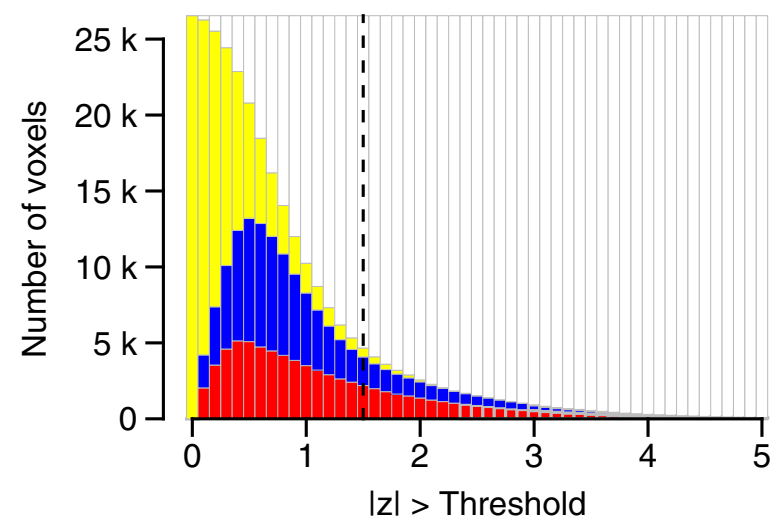

IC3 $>$ thres. \& IC4 > thres.

IC4 $>$ thres. \& IC $3 \leq$ thres.

IC3 $>$ thres. \& IC4 $\leq$ thres.

Both ICs $\leq$ thres.
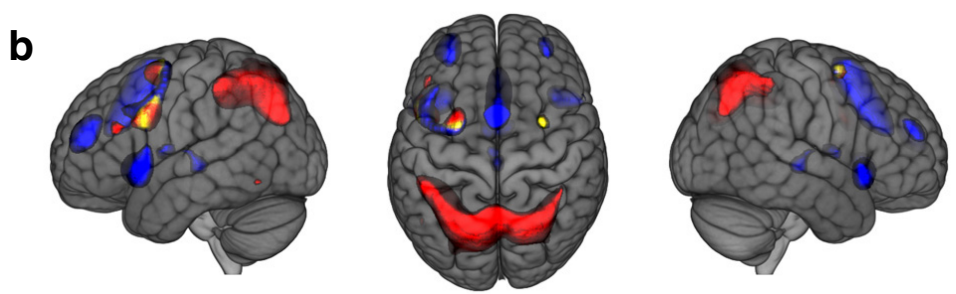

Figure 4. Overlap between WMN-IC3 and WMN-IC4. $\boldsymbol{A}$, The effects of curtailing the voxel loadings of WMN-IC3 and WMN-IC4 using different thresholds ranging from $|z|>0$ to $|z|>5$ (x-axis). Stacked bars $(y$-axis) depict the share of all $N=26542$ voxels that load onto both WMN-IC3 and WMN-IC4 (yellow; i.e., overlap between WMN-IC3 and WMN-IC4), onto WMN-IC4 but not WMN-IC3 (blue), onto WMN-IC3 > thres but not WMN-IC4 $\leq$ thres (red), and onto neither WMN-IC (white) above the threshold indicated by the $x$-axis. The dashed vertical line highlights the share of voxels loading onto the WMN-ICs above a threshold of $|z|>1.47$. This threshold includes the most extreme $10 \%$ of values across all WMN-ICs and was used for illustrating the brain images and to determine the overlap between WMN-IC3 and WMN-IC4 throughout the paper. B, Brain regions loading with $z>1.47$ onto both WMN-IC3 and WMN-IC4 (yellow; i.e., overlap between WMN-IC3 and WMN-IC4), only onto WMN-IC4 (blue), and only onto WMN-IC3 (red). The anatomic annotations of clusters loading onto both WMN-IC3 and WMN-IC4 when considering the most extreme $10 \%$ of loadings are described in Extended data Figure 4-1. The brain images are displayed within the MNI152 template and according to neurologic convention.

tive loadings bilaterally in frontal regions such as the caudal anterior cingulate gyrus, the insula, and the middle frontal gyrus (Fig. 3C; Extended data Fig. 3-1; focusing on the most extreme $10 \%$ of loadings).

The voxel loadings of WMN-IC3 and WMN-IC4 showed only minor overlaps when focusing on the most extreme $10 \%$ of loadings, with $1 \%$ of all WMN-voxels showing $z>$ 1.47 in both WMN-IC3 and WMN-IC4. The overlaps of WMN-IC3 and WMN-IC4 for this threshold, as well as for a range of other thresholds, are illustrated in Figure 4 (yellow color). The overlaps between WMN-IC3 and WMN-IC4 for the most extreme $10 \%$ of loadings comprised three distinct clusters of adjacent voxels (Extended data Fig. 4-1). Two of these clusters were located bilaterally in the middle frontal gyrus and the posterior part of the superior frontal gyrus. The third cluster was located in the left superior parietal cortex.

The voxel loadings of the remaining WMN-ICs are shown in Figure 5. Two components showed predominantly lateralized loadings (WMN-IC5 left, WMN-IC6 right) in frontal regions, inferior parietal regions and the cerebellum when focusing on the most extreme $10 \%$ of loadings. IC6 was associated with episodic memory performance $\left(p_{F D R}=\right.$ $0.0009, R^{2}=0.010$ ); IC5 did not show any FDR-corrected significant associations with task performances. WMNIC2 loaded bilaterally onto occipital regions like the fusiform gyrus and the lingual gyrus, as well as the cerebellum and the thalamus when considering the most extreme $10 \%$ of loadings, and was associated with episodic memory performance $\left(p_{F D R}=0.006, R^{2}=0.006\right)$ and D-prime 0-back performance $\left(p_{F D R}=0.02, R^{2}=0.005\right)$. WMN-IC1 loaded bilaterally onto the precuneus, frontal and inferior parietal regions when focusing on the most extreme $10 \%$ of loadings and did not show any FDR-corrected significant associations with task performances.

In summary, within the WMN, two out of six networks functionally differentiated between WM performance and attention. A parietally-centered network was mainly associated with WM-related performance and a frontallycentered network was mainly associated with attentionrelated performance. We verified these results by applying voxel-wise association analyses between the 2-back - 

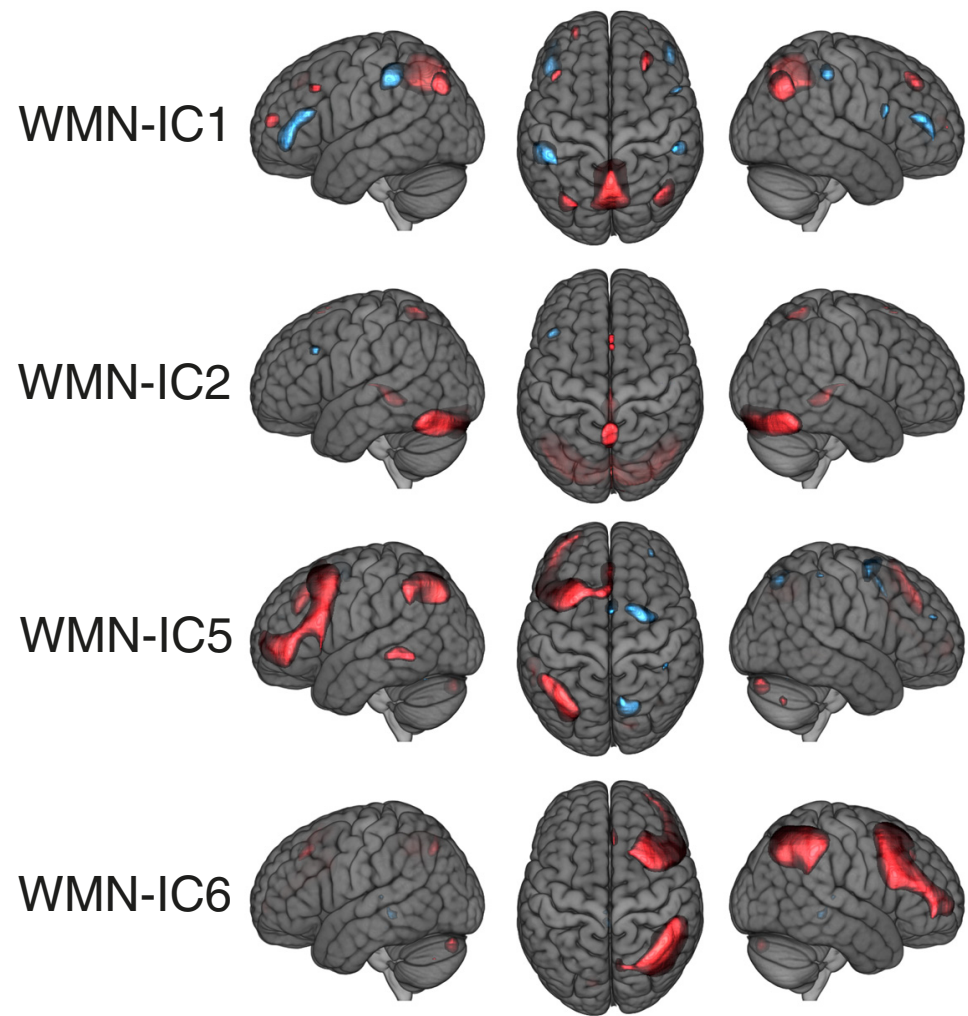

Figure 5. WMN ICA decomposition voxel loadings of the remaining WMN-ICs. The threshold of $|z|>1.47$ used for illustration displays the most extreme $10 \%$ of the voxel loadings across all WMN-ICs; red depicts positive and blue negative voxel loadings. The brain images are displayed within the MNI152 template and according to neurologic convention.

0-back contrast parameter estimates and D-prime 0-back as well as D-prime 2-back performances (Fig. 6A,B; see also " $\beta$ performance 0 -back" and " $\beta$ performance 2-back" in NeuroVault; see Extended data Table 1-2 for the remaining variables). On this voxel-wise level, mainly parietal and superior frontal voxels showed positive associations with D-prime 2-back performance and mainly frontal regions showed positive associations with D-prime 0-back performance.

To confirm the stability of the main results from the ICA we applied bootstrapping and cross-validation procedures. The bootstrapping revealed stable network decomposition and robust associations of these networks with task performances in subsamples of $N=100$ (Fig.

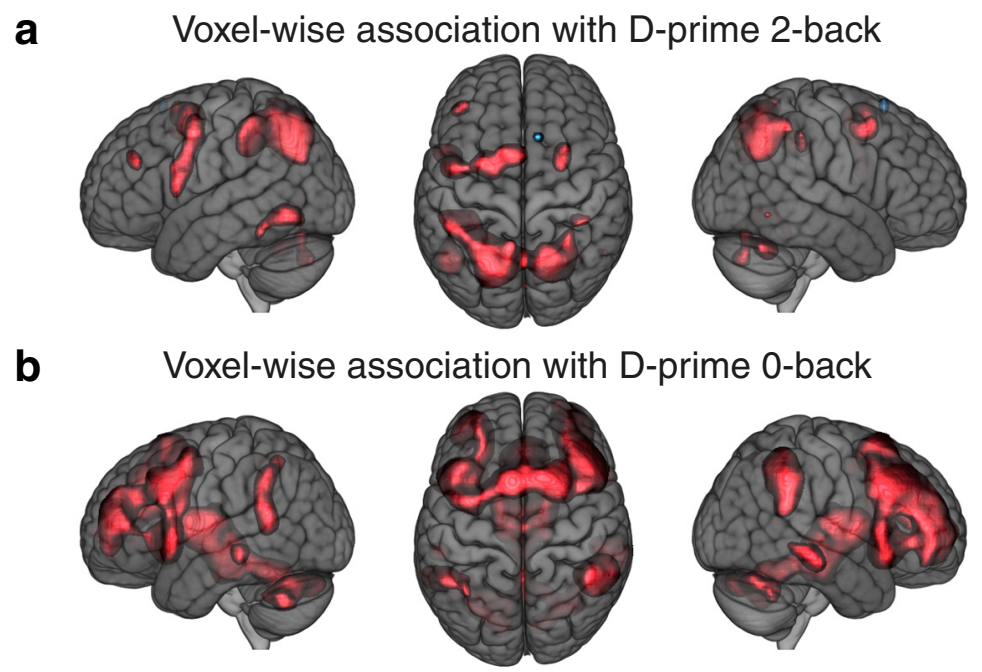

Figure 6. WMN voxel-wise association results. Univariate results for WMN voxels against D-prime 2-back (A) and D-prime 0-back (B) task performances $(N=1369$, $\mathrm{df}=1354)$; red clusters show FDR-corrected significant positive associations. The brain images are displayed within the MNI152 template and according to neurologic convention. 
a

Correlation of subsample voxel loadings

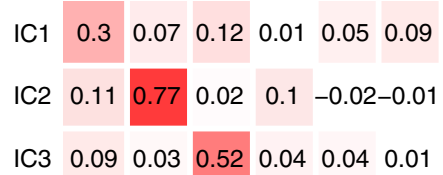

$N=100$

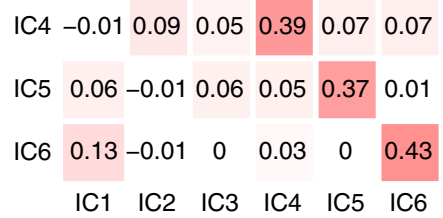

d

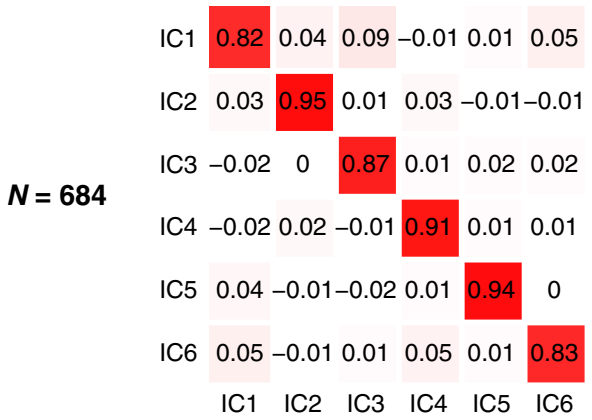

b

Associations with IC3 in subsamples

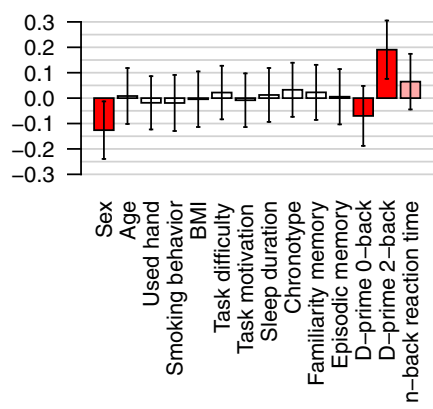

e
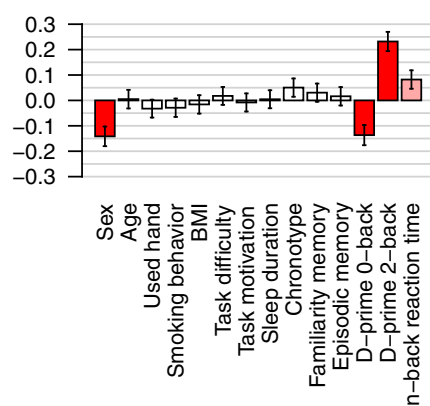

C

Associations with IC4 in subsamples
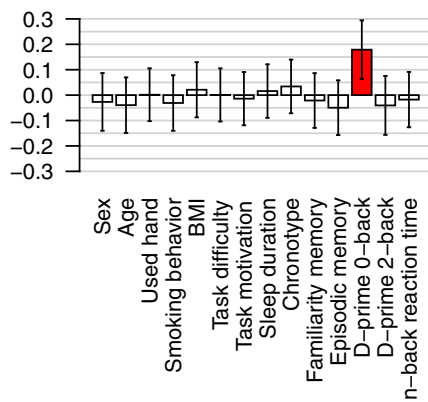

f
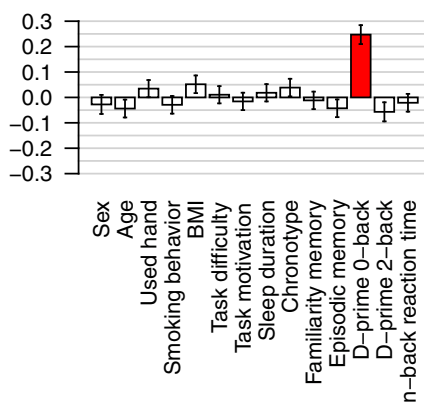

Figure 7. WMN ICA decomposition bootstrapping results. Pearson's correlation coefficients comparing the voxel loadings of ICA decompositions between two nonintersecting subsamples of sizes $(\boldsymbol{A}) N=100$ each and $(\boldsymbol{D}) N=684$ each (i.e., split-halves). Depicted are the averaged correlation coefficients across 100 runs. The associations of WMN-IC 3 with task performances and covariates averaged across the $2 \times 100$ random subsamples are shown for $(\boldsymbol{B}) N=100(\mathrm{df}=85)$ and $(\boldsymbol{E}) N=684(\mathrm{df}=669)$. The associations of WMN-IC4 with task performances and covariates averaged across the $2 \times 100$ random subsamples are shown for $(\boldsymbol{C}) N=100(\mathrm{df}=85)$ and $(\boldsymbol{F}) N=684(\mathrm{df}=669)$. Bars represent the averaged regression coefficients; error bars denote the averaged standard errors of the regression coefficients; red colors in the bar plots describe the FDR-corrected significance of the corresponding WMN-IC's association with the independent variables in the total sample (see top-right legend).

$7 A-C$ ) and of $N=684$ (i.e., split-half; Fig. 7D-F). Crossvalidations additionally demonstrated that ICA solutions estimated in a larger subsample could predict task performance in another nonintersecting smaller subsample (WMNIC3 and D-prime 2-back: averaged $\beta=0.25, p_{\text {nominal }}<0.05$ in $64 \%$ of runs; WMN-IC4 and D-prime 0-back: averaged $\beta=0.23 p_{\text {nominal }}<0.05$ in $61 \%$ of runs; expected under $\mathrm{H}_{0}$ is $5 \%, p_{\text {empirical }}<0.001$ in both analyses). Additionally, we repeated the ICA decomposition and the association analyses with a varying number of extracted components (between 2 and 10). The results remained very similar when using more than three components (Extended data Figs. 3-2, 3-3). The estimated WMN-ICs from the sixcomponents solution are provided in NeuroVault (" $z$ value voxel loadings WMN-IC").

\section{Association of WM-task networks with cortical white matter microstructure}

Differences in cortical white matter microstructure impact the activity in functional brain networks (Andrews-
Hanna et al., 2007; Burzynska et al., 2011; Palacios et al., 2012; Marstaller et al., 2015). We tested for a global association between white matter microstructure and differences in WMN-IC scores in our sample, separately for each WMN-IC. Cortical white matter microstructure was measured by DTI. We used FA values that are related to fiber orientation (Jones et al., 2013). Data were available for 70 white matter-segmented brain regions in a subsample of 614 subjects from our study. Out of the six networks, the parietally-centered network WMN-IC3 showed a significant global association between white matter microstructure and strength of network activation (Kolmogorov-Smirnov: $D=0.37, p_{F D R}=1.6 \times 10^{-8}$, for all remaining WMN-ICs $p_{F D R}>0.24$; Empiric: $p_{F D R}=0.01$, for all remaining WMN-ICs $p_{F D R}>0.32$; Fig. 8). The largest positive associations between $\mathrm{WMN}-\mathrm{IC} 3$ and FA values were found in white matter regions adjacent to the posterior cingulum, the superior parietal cortex, and the precentral gyrus (Table 2). 
a

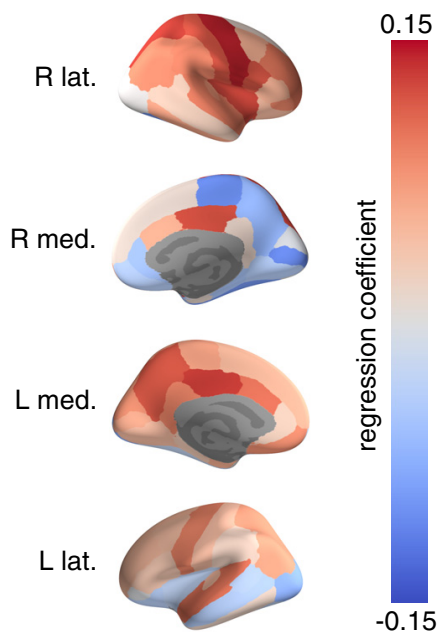

b

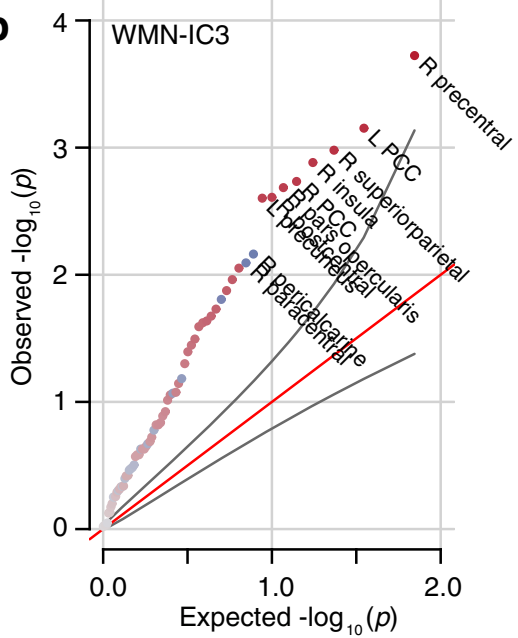

C
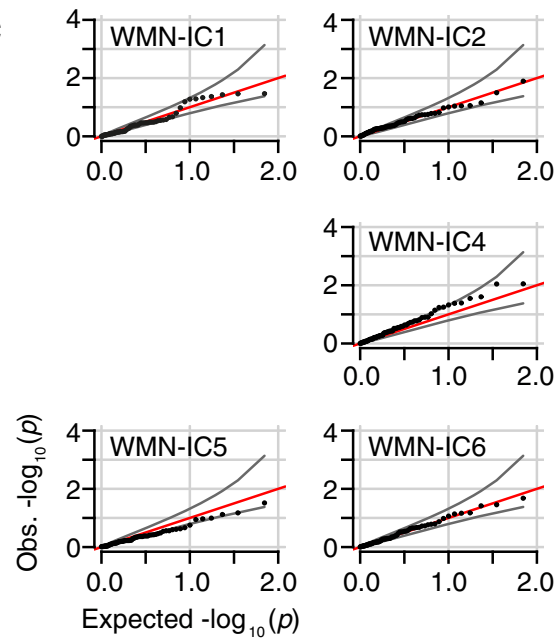

Figure 8. WMN ICA associations with cortical white matter microstructure. A, Associations of WMN-IC3 with the averaged FA values in 70 cortical white matter areas $(N=614, \mathrm{df}=602) . B, \boldsymbol{C}$, Quantile-quantile plots of the -log10 $(p)$ values from the linear regressions of averaged FA values against $(\boldsymbol{B})$ WMN-IC3 and $(\boldsymbol{C})$ the remaining five WMN-ICs. The Quantile-quantile plot compares the distribution of $-\log 10(p)$ values expected at random (x-axis) with the distribution of the observed $-\log 10(p)$ values $(y$-axis). Gray curves indicate 95\% confidence intervals. Detailed results for all 70 areas are listed in Table 2. R: right; L: left; lat.: lateral; med.: medial.

\section{Comparison of the WM-task networks with external datasets}

Functional brain networks can be specifically activated in one given task or can be involved in a variety of different tasks (Cole et al., 2014). To assess the specificity of the WMN-ICs we compared them with results from other studies that cover a wider range of different tasks. We investigated whether the networks derived from the verbal n-back task had previously been identified in others studies using not only the n-back task but also different WM-related paradigms.

We first compared our results with the results of an extensive meta-analysis of neuroimaging studies that includes a number of different WM tasks (Rottschy et al., 2012). The authors reported a "WM core network" of 10 regions that were consistently activated across distinct WM tasks, designs and contrasts. Seven out of these 10 regions overlapped with voxels showing high loadings $(z>1.47)$ in WMN-IC3 or WMN-IC4 derived from our data (Fig. 9): Three of these 10 regions showed high loadings on WMN-IC3 only and three regions showed high loadings on IC4 when focusing on the most extreme $10 \%$ of loadings. One region shared high loadings on both WMNIC3 and WMN-IC4.

Next, we assessed whether the networks identified with our data show similarities with networks derived from NeuroSynth (Yarkoni et al., 2011). NeuroSynth is a metaanalytical brain imaging resource that provides information from $11406 \mathrm{fMRI}$ studies covering a wide range of distinct tasks. Based on a PCA (Fig. 10A) and ICA decomposition, we retrieved 16 global networks of brain activations that were found across the included studies and terms (all estimated networks are described in Table 3, Extended data Fig. 10-1; the estimated NeuroSynth-ICs are additionally provided in NeuroVault " $z$ value voxel loadings NeuroSynth-IC"). Two of these global networks (NeuroSynth IC-topic 11 "DLPFC" and IC-topic 8 "pari- etal") were to a large extent ( $>80 \%$ of the voxels with $z>$ $0.70 ;|z|>0.7$ described the most extreme $10 \%$ of absolute values across the NeuroSynth-ICs) located within the WMN derived from our data (Table 3). We compared the loadings of these two networks with the loadings of WMN-ICs of our data. We then retrieved scores of the two NeuroSynth networks for our subjects and associated them with the subjects' task performances. The parietal network (Fig. 10B) showed a profound similarity with WMN-IC3 (42\% shared variance when comparing voxel loadings within the WMN). The subject-wise scores derived for the NeuroSynth IC-topic parietal were very similar to the scores of our WMN-IC3 $\left(r_{\text {Pearson }}=0.77 ; 59 \%\right.$ shared variance; Fig. 10D). Correspondingly, WM performance also showed a highly significant association with scores derived for the NeuroSynth IC-topic parietal in our sample (D-prime 2-back: $p_{\mathrm{FDR}}=2.4 \times 10^{-10}, R^{2}=0.04$; D-prime 0-back: $\left.p_{F D R}=0.20, R^{2}=0.002\right)$. We did not find a profound similarity of the DLPFC network's voxel loadings (Fig. 10C) with any of our WMN-ICs (shared variances $<3.3 \%$ ). However, the subject-wise scores of the DLPFC network were moderately correlated with the scores of WMN-IC4 $\left(r_{\text {Pearson }}=0.25 ; 6 \%\right.$ shared variance; Fig. 10E) and were also associated with D-prime 0-back performance in our sample (D-prime 0-back: $p_{F D R}=1.6 \times$ $10^{-8}, R^{2}=0.04$; D-prime 2-back: $p_{F D R}=0.45, R^{2}<0.001$ ).

\section{Discussion}

Studies on WM related brain activation typically describe a fronto-parietal network being implicated in WM tasks (Klingberg et al., 2002; Owen et al., 2005; Rottschy et al., 2012; Satterthwaite et al., 2013; Constantinidis and Klingberg, 2016; Huang et al., 2016). Based on ICA decomposition we have identified two networks within the WMN that showed distinct functional characteristics. A network with prominent parietal and smaller frontal features was mainly associated with WM-related perfor- 
Table 2. Associations of WMN-IC 3 with DTI measurements

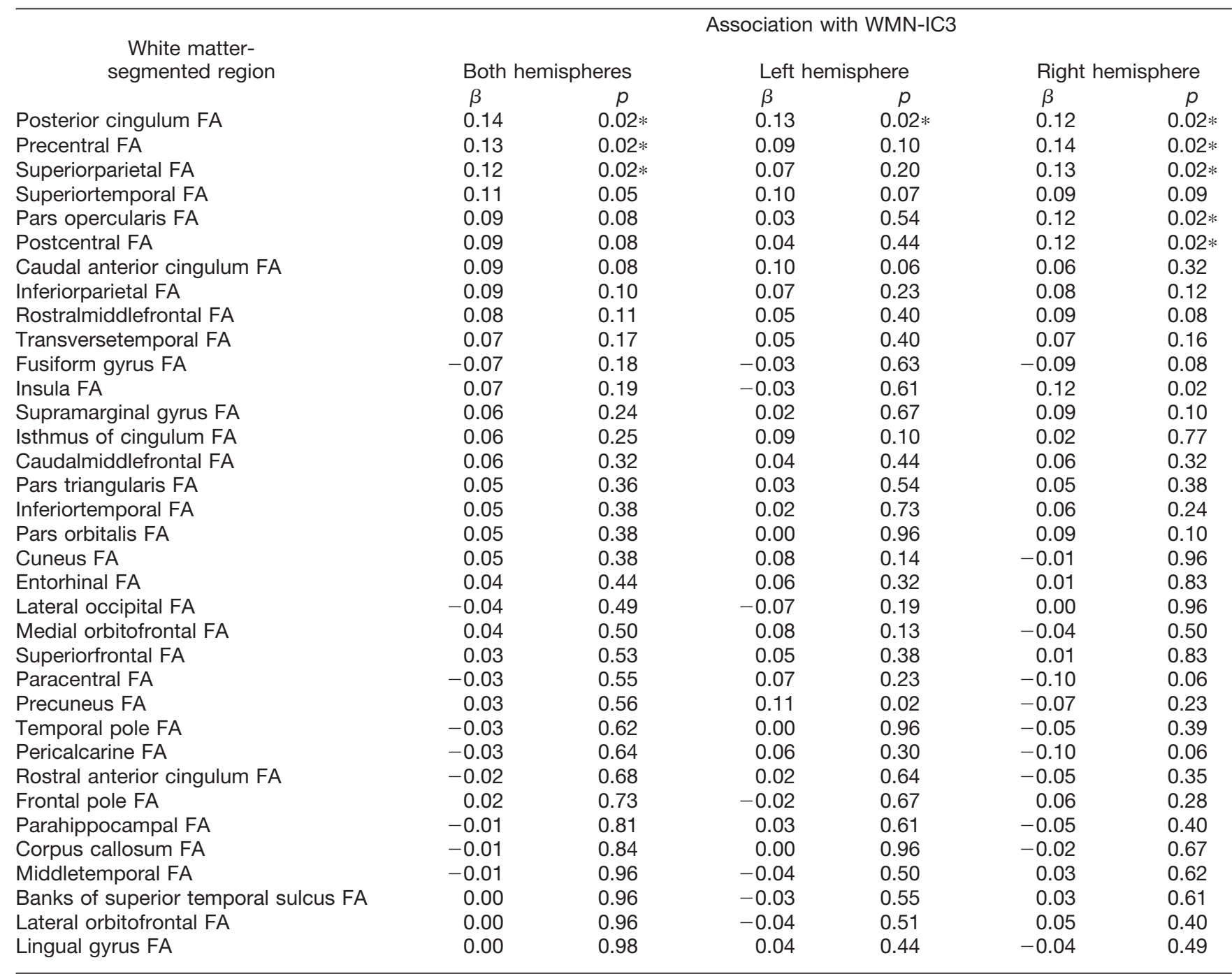

The reported $p$ values are FDR corrected $(\alpha<0.05)$ for three (both hemispheres, left hemisphere, right hemisphere) $\times 70$ (anatomic regions) tests; $* p<0.05$. All df $=602$.

Shown are FDR-corrected $p$ values and regression coefficients describing the associations of FA measures (averaged across both hemispheres, for the left hemisphere, and for the right hemisphere) with the estimates of WMN-IC3.

mance $(5.8 \%$ variance explained), the associations with attention-related performance were smaller $(2.2 \%$ variance explained) and in the opposite direction of effect. A second network of predominantly frontal areas (left DLPFC,
ACC, both insulae) was merely relevant for attention-related behavior (6.2\% variance explained).

Our findings of a frontally-centered and a parietallycentered network involved in different aspects of WM-

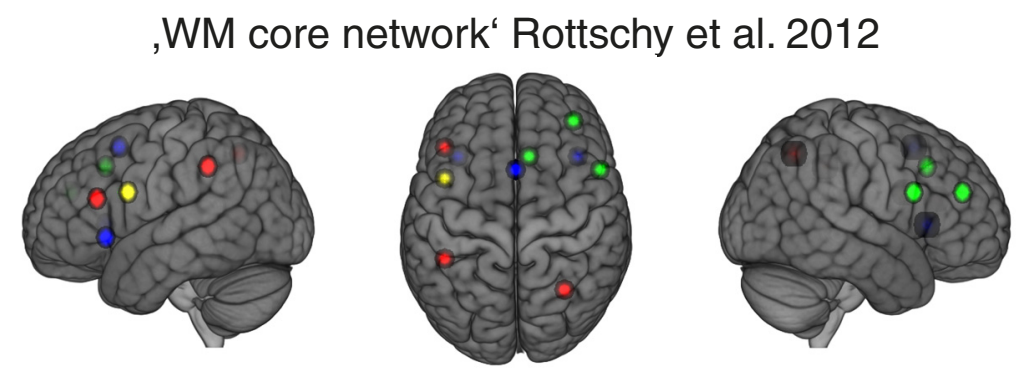

Figure 9. WMN-IC3 and WMN-IC4 in comparison with a WM core network described in Rottschy et al. (2012). Red regions overlap with WMN-IC3 ( $z>1.47)$, blue regions overlap with WMN-IC4 $(z>1.47)$, yellow region overlaps with WMN-IC3 and WMN-IC4; the green regions do not overlap with WMN-IC3 or WMN-IC4. The brain images are displayed within the MNI152 template and according to neurologic convention. 


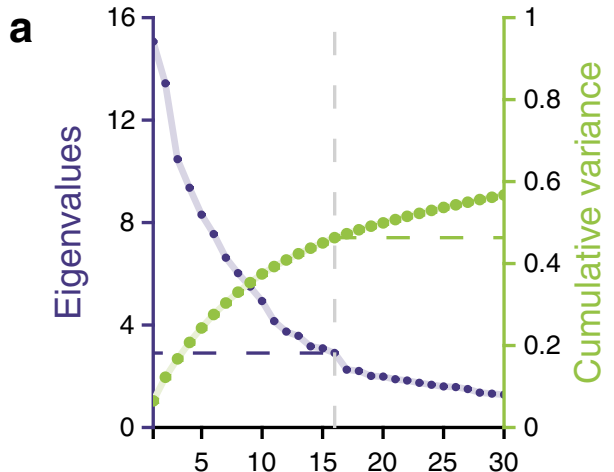

b

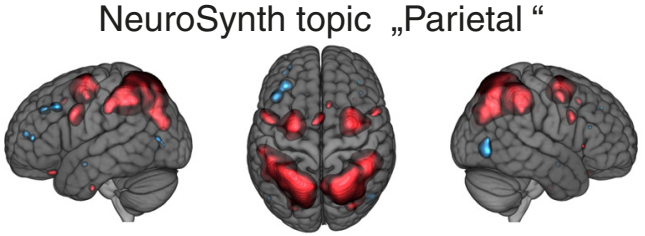

C
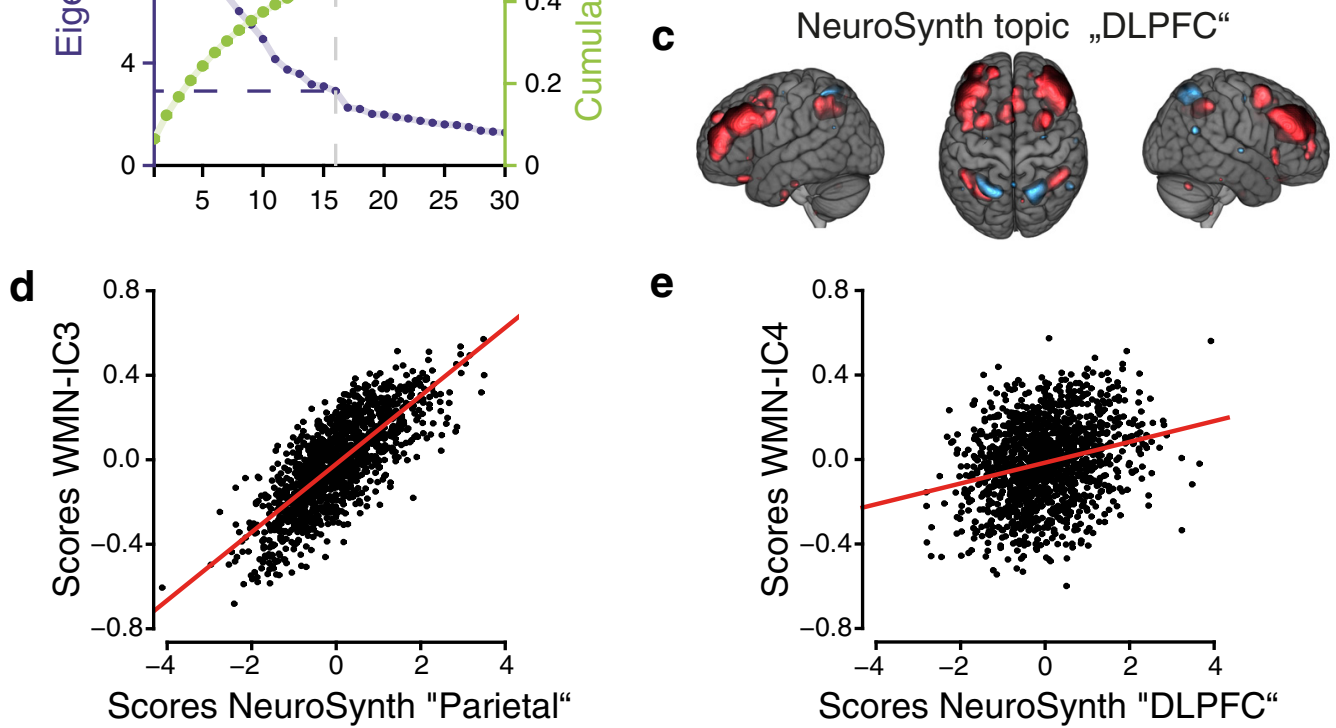

e

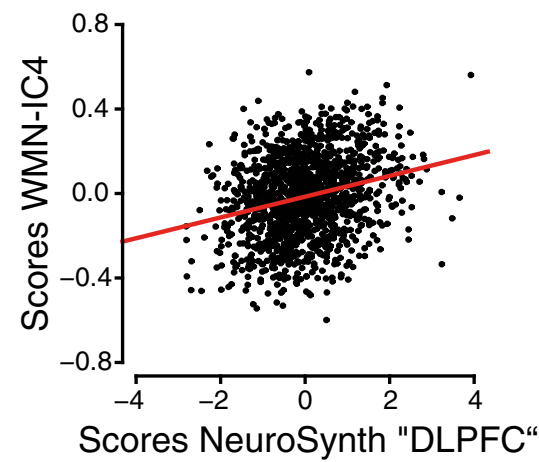

Figure 10. WMN-IC3 and WMN-IC4 compared to an ICA decomposition derived from NeuroSynth. $\boldsymbol{A}$, The eigenvalues (purple, left $y$-axis) and cumulative variance (green, right $y$-axis) of the PCA on voxel loadings of 233 NeuroSynth terms. Voxel loadings (B) of the NeuroSynth IC-topic parietal (IC8) and voxel loadings $(\boldsymbol{C})$ of the NeuroSynth IC-topic DLPFC (IC11) with $|z|>0.70$. D, E, Comparison of the subject-wise scores of the WMN-IC3 and WMN-IC4 with the subject-wise scores of the NeuroSynth IC-topic eight parietal $\left(r_{\text {Pearson }}=0.76\right)$ and IC-topic 11 DLPFC $\left(r_{\text {Pearson }}=0.25\right)$. All 16 estimated NeuroSynth IC-topics are shown in Extended data Figure 10-1. The brain images are displayed within the MNI152 template and according to radiologic convention.

task performances are in line with recent ROI-based studies that have reported distinct functional roles of frontal and parietal regions on WM (Kundu et al., 2015; Sarma et al., 2016; Li et al., 2017). The functional results from our estimated networks were also consistent with the voxel-wise results from our data. Notably, using ICA decomposition to estimate brain networks resulted in several advantages as compared to voxel-wise or ROI-based analyses. Both voxel-wise and ROI-based approaches require prior knowledge for defining brain activation patterns relevant for performance, either regarding the subject's task performance or the anatomic ROls. In contrast, the ICA decompositions applied here estimated brain activation networks based on the WMN contrast estimates and did neither include performance measures into the estimation nor preselect voxels based on prior assumptions. Thus, the WMN-ICs constitute data-driven and unbiased measures of brain networks that underlie the task performances. Importantly, ICA decomposition optimized the detection rate of true effects for associating brain activation with WM task performance by considerably decreasing the number of tests performed, from $N=$ 26,542 voxel-wise tests to 6 association analyses with the WMN-ICs, effectively reducing the false-positive rate and increasing statistical power. Furthermore, ICA decomposition enabled us to represent brain networks that were statistically maximally independent. By using the sub- ject's performance measurements, we could show that these networks exhibit distinct functional characteristics. Subjects with high scores on a WMN-IC showed increased coactivation of the voxels that loaded highly onto this WMN-IC, we thus interpreted WMN-ICs as networks of coactivating brain regions. The identification of distinct functional networks within the WM brain activation is in line with numerous recent studies demonstrating that the brain activation at rest as well as during different tasks is most likely based on distinct but possibly spatially overlapping networks (Power et al., 2011; Cole et al., 2014, 2016; Xu et al., 2016). In contrast, univariate voxel-wise analyses or ROI-based approaches would not allow to identify data-driven and statistically independent subnetworks of brain activation that underlie the brain activation during the WM task. Importantly, due to the large sample size used here we can provide robust network estimates that can also be applied to samples with smaller sample sizes.

Sets of brain regions that appear similar to our parietally-centered network have been described in past studies as orienting system for visual events (Fan and Posner, 2004) or dorsal attention network (Power et al., 2011; Petersen and Posner, 2012). The frontally-centered network derived from our data resembles the cinguloopercular network that has been linked to maintaining alertness (Coste and Kleinschmidt, 2016). The two net- 
Table 3. Description of ICs estimated from NeuroSynth data

\begin{tabular}{|c|c|c|c|}
\hline $\begin{array}{l}\text { NeuroSynth } \\
\text { IC-topic }\end{array}$ & IC\# & The 10 most-contributing NeuroSynth terms & $\begin{array}{l}\% \text { voxels } \\
\text { in WMN }\end{array}$ \\
\hline DLPFC & 11 & $\begin{array}{l}\text { Dorsolateral; dorsolateral_prefrontal; dlpfc; cortex_dlpfc; working; } \\
\text { working_memory; prefrontal; prefrontal_cortex; executive; load }\end{array}$ & $88 \%$ \\
\hline Parietal & 8 & $\begin{array}{l}\text { Intraparietal; intraparietal_sulcus; parietal_cortex; parietal; posterior_parietal; } \\
\text { superior_parietal; spatial; fronto_parietal; attentional; sulcus }\end{array}$ & $83 \%$ \\
\hline $\begin{array}{l}\text { Morphometry } \\
\text { versus demand }\end{array}$ & 9 & $\begin{array}{l}\text { Voxel; matter; morphometry; voxel_morphometry; demands; volume; task; } \\
\text { difficulty; working; working_memory }\end{array}$ & $71 \%$ \\
\hline Inferior frontal & 12 & $\begin{array}{l}\text { Inferior_frontal; semantic; word; inferior; language; frontal_gyrus; words; } \\
\text { sentence; meaning; sentences }\end{array}$ & $59 \%$ \\
\hline Fusiform gyrus & 7 & $\begin{array}{l}\text { Fusiform; fusiform_gyrus; face; objects; faces; recognition; category; object; } \\
\text { visual; occipital }\end{array}$ & $54 \%$ \\
\hline Motion/observation & 13 & $\begin{array}{l}\text { Motion; body; observation; viewed; perception; actions; visual; occipital_cortex; } \\
\text { direction; viewing }\end{array}$ & $52 \%$ \\
\hline Motor cortex & 15 & $\begin{array}{l}\text { Motor; movement; motor_cortex; primary_motor; hand; finger; movements; } \\
\text { premotor; sensorimotor; supplementary_motor }\end{array}$ & $51 \%$ \\
\hline Sensory system & 5 & $\begin{array}{l}\text { Secondary; somatosensory; pain; somatosensory_cortex; stimulation; insular; } \\
\text { insula; primary; sensory; intensity }\end{array}$ & $42 \%$ \\
\hline Basal ganglia & 2 & $\begin{array}{l}\text { Basal_ganglia; ganglia; basal; putamen; subcortical; thalamus; striatal; caudate; } \\
\text { striatum; nucleus }\end{array}$ & $42 \%$ \\
\hline Temporal & 4 & $\begin{array}{l}\text { Superior_temporal; superior; auditory; speech; temporal_gyrus; temporal_sulcus; } \\
\text { temporal; posterior_superior; linguistic; gyrus }\end{array}$ & $40 \%$ \\
\hline ACC & 16 & $\begin{array}{l}\text { Anterior_cingulate; anterior; acc; cingulate_cortex; cingulate; cortex_acc; } \\
\text { dorsal_anterior; anterior_insula; insula; cortex_anterior }\end{array}$ & $40 \%$ \\
\hline Striatum & 10 & $\begin{array}{l}\text { Ventral_striatum; reward; striatum; ventral; value; nucleus; striatal; } \\
\text { decision_making; orbitofrontal; orbitofrontal_cortex }\end{array}$ & $38 \%$ \\
\hline Medial prefrontal & 6 & $\begin{array}{l}\text { Social; medial_prefrontal; junction; theory; temporo; medial; states; person; } \\
\text { mental; prefrontal_cortex }\end{array}$ & $35 \%$ \\
\hline Default mode & 3 & $\begin{array}{l}\text { Default_mode; mode; default; mode_network; resting; resting_state; state; } \\
\text { posterior_cingulate; independent_component; functional_connectivity }\end{array}$ & $33 \%$ \\
\hline Hippocampus & 14 & $\begin{array}{l}\text { Hippocampal; medial_temporal; hippocampus; parahippocampal; temporal_lobe; } \\
\text { encoding; episodic; episodic_memory; parahippocampal_gyrus; lobe }\end{array}$ & $20 \%$ \\
\hline Amygdala & 1 & $\begin{array}{l}\text { Neutral; amygdala; emotion; fear; emotional; expressions; facial; affective; } \\
\text { emotions; anxiety }\end{array}$ & $14 \%$ \\
\hline
\end{tabular}

IC-topics were assigned based on NeuroSynth terms with the highest loading. For voxels showing the highest loadings $(z>0.70)$, we calculated the percentage of voxels being located within the WMN (\% voxels in WMN).

works identified in our study spatially overlap in three separate clusters when focusing on the most extreme $10 \%$ of loadings. Two of these clusters were located bilaterally in the middle frontal gyrus and the posterior part of the superior frontal gyrus. The third cluster was located in the left superior parietal cortex. Overlaps between brain networks could represent regions of convergence between otherwise segregated functional networks (Sporns, 2013). Links between distinct networks are presumably features of brain organization and important for complex behaviors (Yeo et al., 2014). Accordingly, the lateral PFC (which includes the middle frontal gyrus) has been proposed to serve as a globally connected functional hub that is involved in cognitive control (Cole et al., 2012). Together, the most extreme $10 \%$ of voxel loadings of the two networks relevant for WM task performance in our study closely overlap with a WM core network identified in an extensive meta-analysis of WM neuroimaging studies by Rottschy et al. (2012) that included a number of other WM tasks besides the verbal n-back task used here. Importantly, both of the two networks estimated in our study overlap with distinct parts of this global WM core network. Furthermore, the parietally-centered network identified in our study sample showed considerable similarity with a parietal network derived from NeuroSynth
(Yarkoni et al., 2011). This parietal network derived from NeuroSynth was estimated across a large body of results from neuroimaging studies using many different paradigms. These results imply that especially the parietallycentered network, which was associated with WM-related task performance in our sample, is an important and stable network implicated in WM-related cognitive functioning.

This parietally-centered network was furthermore associated with global differences in FA estimates in our subjects. FA describes aspects of white matter microstructure related to fiber orientation (Jones et al., 2013) and can be modulated by myelination (Beaulieu, 2002). Measurements of FA have been observed to decrease with increasing age (Inano et al., 2011) and after moderate to severe traumatic brain injury (Kraus et al., 2007). Properties of white matter microstructure have also been shown to affect large-scale functional networks such as the default mode network (Andrews-Hanna et al., 2007), the WMN (Burzynska et al., 2011; Palacios et al., 2012; Darki and Klingberg, 2015), the salience network, and the fronto-parietal network (Marstaller et al., 2015). The parietally-centered network in our study was globally associated with FA measures across the white mattersegmented regions. Conversely, the other networks esti- 
mated here did not show any FA-associations. Positive associations of FA with $\mathrm{fMRI}$ measurements or with connectivity measures have been proposed to represent better transmission and stronger functional connections (Warbrick et al., 2017). FA measures in fronto-parietal tracts have moreover been associated with WM performance (Charlton et al., 2010; Vestergaard et al., 2011; Darki and Klingberg, 2015). A recent large-scale study of $N=1584$ subjects reported that functional connectivity between brain regions was influenced by lesions in white matter tracts directly connecting the brain regions, but also by white matter load in other, not directly connected tracts (Langen et al., 2017). Thus, global white matter integrity might contribute to the WM performance-relevant coactivation observed in our study. Additionally, we observed that FA measures of single white mattersegmented regions adjacent to the parietally-centered network's cortical main foci (specifically the posterior cingulum, superior parietal, and precentral regions) were associated with coactivation within the network.

WM and attention are closely related neurocognitive domains (Eriksson et al., 2015; Constantinidis and Klingberg, 2016). Importantly, these neurocognitive domains are also affected in neuropsychiatric disorders like schizophrenia (Barch and Ceaser, 2012). A meta-analysis across 41 neuroimaging studies observed reduced activation of the left DLPFC and the ACC in schizophrenia patients during executive tasks (Minzenberg et al., 2009). Barch and Ceaser (2012) depicted that the robustly observed altered DLPFC activation in schizophrenia could either directly impact cognitive functions such as WM or interfere with top-down functions such as proactive control that in turn mediate the effect on WM. Our observation that a network of frontal regions including the DLPFC and ACC was mainly associated with attention-related performance coincides with the assumption of impaired general executive functions rather than isolated WM function in schizophrenia. Other studies investigating cognitive deficits in schizophrenia have come to similar conclusions of a deficit in general cognitive ability in schizophrenia (Haut et al., 2015).

To summarize, we have identified two networks within the WMN that showed distinct functional characteristics with respect to attention-related and WM-related task performances. Compared to voxel-wise analyses, using a multivariate approach led to more specific results with higher effect sizes and higher statistical power while minimizing the burden of multiple testing. Low statistical power in combination with a large number of statistical tests is a prevalent source of critique regarding the existing neuroimaging literature (Poldrack et al., 2017; Szucs and loannidis, 2017), especially in combination with multiple high-dimensional datasets such as imaging genetic studies (Bigos and Weinberger, 2010; Medland et al., 2014; Poline et al., 2015). Van Snellenberg et al. (2016) have stressed that finding replicable biomarkers of WM will help to broaden our understanding of the associated neural, molecular or genetic mechanisms. Our findings take a step in this direction by providing stable network estimates for application in independent samples (http://
neurovault.org/collections/EYCSLZUZ/). This allows future studies to investigate functional distinct brain networks that are implicated in human cognition.

\section{References}

Alderson RM, Kasper LJ, Hudec KL, Patros CHG (2013) Attentiondeficit/hyperactivity disorder (ADHD) and working memory in adults: a meta-analytic review. Neuropsychology 27:287-302. CrossRef

Andrews-Hanna JR, Snyder AZ, Vincent JL, Lustig C, Head D, Raichle ME, Buckner RL (2007) Disruption of large-scale brain systems in advanced aging. Neuron 56:924-935. CrossRef Medline

Ashburner J (2007) A fast diffeomorphic image registration algorithm. Neuroimage 38:95-113. CrossRef Medline

Baddeley A (2012) Working memory: theories, models, and controversies. Annu Rev Psychol 63:1-29. CrossRef Medline

Barch DM, Ceaser A (2012) Cognition in schizophrenia: core psychological and neural mechanisms. Trends Cogn Sci 16:27-34. CrossRef Medline

Basser PJ (1995) Inferring microstructural features and the physiological state of tissues from diffusion-weighted images. NMR Biomed 8:333-344. Medline

Beaulieu C (2002) The basis of anisotropic water diffusion in the nervous system - a technical review. NMR Biomed 15:435-455. CrossRef Medline

Bigos KL, Weinberger DR (2010) Imaging genetics - days of future past. Neuroimage 53:804-809. CrossRef Medline

Burzynska AZ, Nagel IE, Preuschhof C, Li S-C, Lindenberger U, Bäckman L, Heekeren HR (2011) Microstructure of frontoparietal connections predicts cortical responsivity and working memory performance. Cereb Cortex 21:2261-2271. CrossRef Medline

Charlton RA, Barrick TR, Lawes INC, Markus HS, Morris RG (2010) White matter pathways associated with working memory in normal aging. Cortex 46:474-489. CrossRef

Chiappetta P, Roubaud MC, Torrésani B (2004) Blind source separation and the analysis of microarray data. J Comput Biol 11:10901109. CrossRef Medline

Cole MW, Yarkoni T, Repovs G, Anticevic A, Braver TS (2012) Global connectivity of prefrontal cortex predicts cognitive control and intelligence. J Neurosci 32:8988-8999. CrossRef Medline

Cole MW, Bassett DS, Power JD, Braver TS, Petersen SE (2014) Intrinsic and task-evoked network architectures of the human brain. Neuron 83:238-251. CrossRef Medline

Cole MW, Ito T, Bassett DS, Schultz DH (2016) Activity flow over resting-state networks shapes cognitive task activations. Nat Neurosci 19:1718-1726. CrossRef

Constantinidis C, Klingberg T (2016) The neuroscience of working memory capacity and training. Nat Rev Neurosci 17:438-449. CrossRef Medline

Coste CP, Kleinschmidt A (2016) Cingulo-opercular network activity maintains alertness. Neuroimage 128:264-272. CrossRef Medline

Darki F, Klingberg T (2015) The role of fronto-parietal and frontostriatal networks in the development of working memory: a longitudinal study. Cereb Cortex 25:1587-1595. CrossRef Medline

Desikan RS, Ségonne F, Fischl B, Quinn BT, Dickerson BC, Blacker D, Buckner RL, Dale AM, Maguire RP, Hyman BT, Albert MS, Killiany RJ (2006) An automated labeling system for subdividing the human cerebral cortex on MRI scans into gyral based regions of interest. Neuroimage 31:968-980. CrossRef Medline

Engreitz JM, Daigle BJ, Marshall JJ, Altman RB (2010) Independent component analysis: mining microarray data for fundamental human gene expression modules. J Biomed Inform 43:932-944 CrossRef Medline

Eriksson J, Vogel EK, Lansner A, Bergström F, Nyberg L (2015) Neurocognitive architecture of working memory. Neuron 88:3346. CrossRef Medline

Fan J, Posner M (2004) Human attentional networks. Psychiatr Prax 31:210-214. CrossRef 
Fischl B (2012) FreeSurfer. Neuroimage 62:774-781. CrossRef Medline

Fischl B, Salat DH, Busa E, Albert M, Dieterich M, Haselgrove C, Van der Kouwe A, Killiany R, Kennedy D, Klaveness S, Montillo A, Makris N, Rosen B, Dale AM (2002) Whole brain segmentation: neurotechnique automated labeling of neuroanatomical structures in the human brain. Neuron 33:341-355. CrossRef

Forbes NF, Carrick LA, Mclntosh AM, Lawrie SM (2009) Working memory in schizophrenia: a meta-analysis. Psychol Med 39:889905. CrossRef Medline

Gorgolewski KJ, Varoquaux G, Rivera G, Schwartz Y, Ghosh SS, Maumet C, Sochat VV, Nichols TE, Poldrack RA, Poline J-B, Yarkoni T, Margulies DS (2016) NeuroVault.org: a repository for sharing unthresholded statistical maps, parcellations, and atlases of the human brain. Neuroimage 124:1242-1244. CrossRef Medline

Haut KM, Karlsgodt KH, Bilder RM, Congdon E, Freimer NB, London ED, Sabb FW, Ventura J, Cannon TD (2015) Memory systems in schizophrenia: modularity is preserved but deficits are generalized. Schizophr Res 168:223-230. CrossRef Medline

Heck A, Fastenrath M, Ackermann S, Auschra B, Bickel H, Coynel D, Gschwind L, Jessen F, Kaduszkiewicz H, Maier W, Milnik A, Pentzek M, Riedel-Heller SG, Ripke S, Spalek K, Sullivan P, Vogler C, Wagner M, Weyerer S, Wolfsgruber S, de Quervain DJ-F, Papassotiropoulos A (2014) Converging genetic and functional brain imaging evidence links neuronal excitability to working memory, psychiatric disease, and brain activity. Neuron 81:1203-1213. CrossRef Medline

Huang AS, Klein DN, Leung H-C (2016) Load-related brain activation predicts spatial working memory performance in youth aged 9-12 and is associated with executive function at earlier ages. Dev Cogn Neurosci 17:1-9. CrossRef Medline

Hyvärinen A, Oja E (2000) Independent component analysis: algorithms and applications. Neural Netw 13:411-430. Medline

Inano S, Takao H, Hayashi N, Abe O, Ohtomo K (2011) Effects of age and gender on white matter integrity. Am J Neuroradiol 32:21032109. CrossRef Medline

Jenkinson M, Beckmann CF, Behrens TEJ, Woolrich MW, Smith SM (2012) FSL. Neuroimage 62:782-790. CrossRef

Jones DK, Knösche TR, Turner R (2013) White matter integrity, fiber count, and other fallacies: the do's and don'ts of diffusion MRI. Neuroimage 73:239-254. CrossRef Medline

Klein A, Andersson J, Ardekani BA, Ashburner J, Avants B, Chiang M-C, Christensen GE, Collins DL, Gee J, Hellier P, Song JH, Jenkinson M, Lepage C, Rueckert D, Thompson P, Vercauteren T, Woods RP, Mann JJ, Parsey RV (2009) Evaluation of 14 nonlinear deformation algorithms applied to human brain MRI registration. Neuroimage 46:786-802. CrossRef Medline

Klingberg T, Forssberg $H$, Westerberg $H$ (2002) Increased brain activity in frontal and parietal cortex underlies the development of visuospatial working memory capacity during childhood. J Cogn Neurosci 14:1-10. CrossRef Medline

Kong W, Vanderburg CR, Gunshin H, Rogers JT, Huang X (2008) A review of independent component analysis application to microarray gene expression data. Biotechniques 45:501-520. CrossRef Medline

Kraus MF, Susmaras T, Caughlin BP, Walker CJ, Sweeney JA, Little DM (2007) White matter integrity and cognition in chronic traumatic brain injury: a diffusion tensor imaging study. Brain 130: 2508-2519. CrossRef Medline

Kundu B, Chang J-Y, Postle BR, Van Veen BD (2015) Contextspecific differences in fronto-parieto-occipital effective connectivity during short-term memory maintenance. Neuroimage 114:320327. CrossRef Medline

Lang PJ, Bradley MM, Cuthbert BN (2008) International affective picture system (IAPS): affective ratings of pictures and instruction manual. Technical report A-8. Gainesville, FL: University of Florida. Available at http://www.unifesp.br/dpsicobio/adap/instructions.pdf.
Langen CD, Zonneveld HI, White T, Huizinga W, Cremers LGM, de Groot M, Ikram MA, Niessen WJ, Vernooij MW (2017) White matter lesions relate to tract-specific reductions in functional connectivity. Neurobiol Aging 51:97-103. CrossRef Medline

Lee J, Park S (2005) Working memory impairments in schizophrenia: a meta-analysis. J Abnorm Psychol 114:599-611. CrossRef Medline

Li S, Cai Y, Liu J, Li D, Feng Z, Chen C, Xue G (2017) Dissociated roles of the parietal and frontal cortices in the scope and control of attention during visual working memory. Neuroimage 149:210219. CrossRef Medline

Macmillan NA, Creelman CD (1990) Response bias: characteristics of detection theory, threshold theory, and "nonparametric" indexes. Psychol Bull 107:401-413. CrossRef

Marazziti D, Consoli G, Picchetti M, Carlini M, Faravelli L (2010) Cognitive impairment in major depression. Eur $\mathrm{J}$ Pharmacol 626: 83-86. CrossRef Medline

Marstaller L, Williams M, Rich A, Savage G, Burianová H (2015) Aging and large-scale functional networks: white matter integrity, gray matter volume, and functional connectivity in the resting state. Neuroscience 290:369-378. CrossRef Medline

Medland SE, Jahanshad N, Neale BM, Thompson PM (2014) Wholegenome analyses of whole-brain data: working within an expanded search space. Nat Neurosci 17:791-800. CrossRef Medline

Mesholam-Gately RI, Giuliano AJ, Goff KP, Faraone SV, Seidman LJ (2009) Neurocognition in first-episode schizophrenia: a metaanalytic review. Neuropsychology 23:315-336. CrossRef Medline

Miller KM, Price CC, Okun MS, Montijo H, Bowers D (2009) Is the $\mathrm{N}$-back task a valid neuropsychological measure for assessing working memory? Arch Clin Neuropsychol 24:711-717. CrossRef Medline

Minzenberg MJ, Laird AR, Thelen S, Carter CS, Glahn DC (2009) Meta-analysis of 41 functional neuroimaging studies of executive function in schizophrenia. Arch Gen Psychiatry 66:811-822. CrossRef

Modat M, Ridgway GR, Taylor ZA, Lehmann M, Barnes J, Hawkes DJ, Fox NC, Ourselin S (2010) Fast free-form deformation using graphics processing units. Comput Methods Programs Biomed 98:278-284. CrossRef Medline

Murdoch DJ, Tsai Y-L, Adcock J (2008) P-values are random variables. Am Stat 62:242-245. CrossRef

Muschelli J, Sweeney E, Lindquist M, Crainiceanu C (2015) fslr: connecting the FSL software with R. R J 7:163-175. Medline

Nagel BJ, Barlett VC, Schweinsburg AD, Tapert SF (2005) Neuropsychological predictors of BOLD response during a spatial working memory task in adolescents: what can performance tell us about fMRI response patterns? J Clin Exp Neuropsychol 27:823839. CrossRef Medline

Owen AM, McMillan KM, Laird AR, Bullmore E (2005) N-back working memory paradigm: a meta-analysis of normative functional neuroimaging studies. Hum Brain Mapp 25:46-59. CrossRef Medline

Palacios EM, Sala-Llonch R, Junque C, Roig T, Tormos JM, Bargallo $N$, Vendrell $P(2012)$ White matter integrity related to functional working memory networks in traumatic brain injury. Neurology 78:852-860. CrossRef Medline

Petersen SE, Posner MI (2012) The attention system of the human brain: 20 years after. Annu Rev Neurosci 35:73-89. CrossRef Medline

Poldrack RA, Baker Cl, Durnez J, Gorgolewski KJ, Matthews PM, Munafò MR, Nichols TE, Poline J-B, Vul E, Yarkoni T (2017) Scanning the horizon: towards transparent and reproducible neuroimaging research. Nat Rev Neurosci 18:115-126. CrossRef Medline

Poline J-B, Breeze JL, Frouin V (2015) Imaging Genetics with fMRI. In: fMRI: from nuclear spins to brain functions (Uludag L, Ugurbil K, Berliner L, eds), pp. 699-738 New York, NY: Springer.

Power JD, Cohen AL, Nelson SM, Wig GS, Barnes KA, Church JA, Vogel AC, Laumann TO, Miezin FM, Schlaggar BL, Petersen SE 
(2011) Functional network organization of the human brain. Neuron 72:665-678. CrossRef Medline

Rottschy C, Langner R, Dogan I, Reetz K, Laird AR, Schulz JB, Fox PT, Eickhoff SB (2012) Modelling neural correlates of working memory: a coordinate-based meta-analysis. Neuroimage 60:830846. CrossRef Medline

Sarma A, Masse NY, Wang X-J, Freedman DJ (2016) Task-specific versus generalized mnemonic representations in parietal and prefrontal cortices. Nat Neurosci 19:143-149. CrossRef

Satterthwaite TD, Wolf DH, Erus G, Ruparel K, Elliott MA, Gennatas ED, Hopson R, Jackson C, Prabhakaran K, Bilker WB, Calkins ME, Loughead J, Smith A, Roalf DR, Hakonarson H, Verma R, Davatzikos C, Gur RC, Gur RE (2013) Functional maturation of the executive system during adolescence. J Neurosci 33:1624916261. CrossRef Medline

Seeley WW, Crawford RK, Zhou J, Miller BL, Greicius MD (2009) Neurodegenerative diseases target large-scale human brain networks. Neuron 62:42-52. CrossRef Medline

Sporns O (2013) Network attributes for segregation and integration in the human brain. Curr Opin Neurobiol 23:162-171. CrossRef Medline

Szucs D, loannidis JPA (2017) Empirical assessment of published effect sizes and power in the recent cognitive neuroscience and psychology literature. PLoS Biol 15:e2000797. CrossRef

Todd JJ, Marois R (2004) Capacity limit of visual short-term memory in human posterior parietal cortex. Nature 428:751-754. CrossRef Medline

Todd JJ, Marois R (2005) Posterior parietal cortex activity predicts individual differences in visual short-term memory capacity. Cogn Affect Behav Neurosci 5:144-155. Medline

Ullman H, Almeida R, Klingberg T (2014) Structural maturation and brain activity predict future working memory capacity during childhood development. J Neurosci 34:1592-1598. CrossRef Medline

Van Snellenberg JX, Girgis RR, Horga G, van de Giessen E, Slifstein M, Ojeil N, Weinstein JJ, Moore H, Lieberman JA, Shohamy D, Smith EE, Abi-Dargham A (2016) Mechanisms of working memory impairment in schizophrenia. Biol Psychiatry 80:617-626. CrossRef Medline

Vestergaard M, Madsen KS, Baaré WFC, Skimminge A, Ejersbo LR, Ramsøy TZ, Gerlach C, Åkeson P, Paulson OB, Jernigan TL (2011) White matter microstructure in superior longitudinal fasciculus associated with spatial working memory performance in children. J Cogn Neurosci 23:2135-2146. CrossRef

Wager TD, Smith EE (2003) Neuroimaging studies of working memory: a meta-analysis. Cogn Affect Behav Neurosci 3:255-274. Medline

Warbrick T, Rosenberg J, Shah NJ (2017) The relationship between BOLD fMRI response and the underlying white matter as measured by fractional anisotropy (FA): a systematic review. Neuroimage 153:369-381. CrossRef Medline

Whitcher B, Schmid VJ, Thornton A (2011) Working with the DICOM and NIfTI data standards in R. J Stat Softw 44:1-28. CrossRef

Xu J, Potenza MN, Calhoun VD, Zhang R, Yip SW, Wall JT, Pearlson GD, Worhunsky PD, Garrison KA, Moran JM (2016) Large-scale functional network overlap is a general property of brain functional organization: reconciling inconsistent $\mathrm{fMRl}$ findings from generallinear-model-based analyses. Neurosci Biobehav Rev 71:83-100. CrossRef Medline

Yarkoni T, Poldrack RA, Nichols TE, Van Essen DC, Wager TD (2011) Large-scale automated synthesis of human functional neuroimaging data. Nat Methods 8:665-670. CrossRef Medline

Yeo BTT, Krienen FM, Chee MWL, Buckner RL (2014) Estimates of segregation and overlap of functional connectivity networks in the human cerebral cortex. Neuroimage 88:212-227. CrossRef

Zhou J, Gennatas ED, Kramer JH, Miller BL, Seeley WW (2012) Predicting regional neurodegeneration from the healthy brain functional connectome. Neuron 73:1216-1227. CrossRef Medline

Zou Q, Ross TJ, Gu H, Geng X, Zuo X-N, Hong LE, Gao J-H, Stein EA, Zang $Y-F$, Yang $Y(2013)$ Intrinsic resting-state activity predicts working memory brain activation and behavioral performance. Hum Brain Mapp 34:3204-3215. CrossRef Medline 